\title{
Interference effect in lepton number violating and conserving meson decays for a left-right symmetric model
}

\author{
Rohini M. Godbole, ${ }^{1, *}$ Siddharth P. Maharathy $\odot,{ }^{2,3, \dagger}$ Sanjoy Mandal $\odot,{ }^{4, \ddagger}$ Manimala Mitra, ${ }^{2,3, \S}$ and Nita Sinha ${ }^{3,5, \|}$ \\ ${ }^{1}$ Centre for High Energy Physics, Indian Institute of Science, Bengaluru-560012, India \\ ${ }^{2}$ Institute of Physics, Sachivalaya Marg, Bhubaneswar 751005, India \\ ${ }^{3}$ Homi Bhabha National Institute, BARC Training School Complex, Anushakti Nagar, \\ Mumbai 400094, India \\ ${ }^{4}$ AHEP Group, Institut de Física Corpuscular, CSIC/Universitat de València, \\ Parc Científic de Paterna. C/ Catedrático José Beltrán, 2 E-46980 Paterna (Valencia), Spain \\ ${ }^{5}$ The Institute of Mathematical Sciences, C.I.T Campus, Taramani, Chennai 600 113, India
}

(Received 19 April 2021; accepted 14 September 2021; published 11 November 2021)

\begin{abstract}
We study the effect of interference on the lepton number violating (LNV) and lepton number conserving (LNC) three-body meson decays $M_{1}^{+} \rightarrow l_{i}^{+} l_{j}^{ \pm} \pi^{\mp}$ that arise in a TeV-scale left-right symmetric model (LRSM) with degenerate or nearly degenerate right-handed (RH) neutrinos. The LRSM contains three RH neutrinos and a RH gauge boson. The RH neutrinos with masses in the range of $M_{N} \sim(\mathrm{MeV}-\mathrm{few} \mathrm{GeV})$ can give resonant enhancement in the semileptonic LNV and LNC meson decays. In the case where only one RH neutrino contributes to these decays, the predicted new physics branching ratios of semileptonic LNV and LNC meson decays $M_{1}^{+} \rightarrow l_{i}^{+} l_{j}^{+} \pi^{-}$and $M_{1}^{+} \rightarrow l_{i}^{+} l_{j}^{-} \pi^{+}$are equal. We find that with at least two RH neutrinos contributing to the process, the LNV and LNC decay rates can differ. Depending on the neutrino mixing angles and $C P$-violating phases, the branching ratios of LNV and LNC decay channels mediated by the heavy neutrinos can be either enhanced or suppressed, and the ratio of these two rates can differ from unity.
\end{abstract}

DOI: 10.1103/PhysRevD.104.095009

\section{INTRODUCTION}

The observation of light neutrino masses and mixings clearly indicates the existence of beyond-the-StandardModel (BSM) physics. A number of models exist in the literature that have been successful in explaining small neutrino masses and their mixings. One of the most interesting models among them is the left-right symmetric model (LRSM) [1], which not only explains the small light neutrino masses but also addresses parity violation in the SM. The model contains three right-handed $(\mathrm{RH})$ neutrinos and two Higgs triplet fields that generate the Majorana mass terms for light neutrinos via the type-I and type-II seesaw mechanisms. The RH neutrinos in this model are Majorana in nature. The Majorana masses violate lepton number, and hence these neutrinos can directly induce LNV processes.

\footnotetext{
*rohini@iisc.ac.in

†siddharth.m@iopb.res.in

*mandal@ific.uv.es

\$manimala@iopb.res.in

nita@imsc.res.in
}

Published by the American Physical Society under the terms of the Creative Commons Attribution 4.0 International license. Further distribution of this work must maintain attribution to the author(s) and the published article's title, journal citation, and DOI. Funded by SCOAP .
The Majorana nature of the light and heavy neutrinos can be tested via the lepton number violating (LNV) neutrinoless double beta decay $(0 \nu \beta \beta)[2-7]$. Their LNV nature can also be probed at the colliders through direct searches [8-13], as well as through the rare LNV decays of mesons and the tau lepton [14-25]. The $\sqrt{s}=13 \mathrm{TeV}$ LHC search in the samesign dilepton and dijet channel has so far ruled out RH neutrino masses in the mass range from $M_{N} \sim 100 \mathrm{GeV}$ up to a few TeV, and $M_{W_{R}}<4.7 \mathrm{TeV}$ [26,27]. The boosted RH neutrino search for the LRSM also places strong constraints on the RH gauge boson mass $M_{W_{R}}>4.8 \mathrm{TeV}[10,28]$ for the $\mathrm{RH}$ neutrino and a $\mathrm{RH}$ gauge boson mass hierarchy of $\mathcal{O}(0.1)$. The nature of couplings of additional charged gauge bosons with leptons has been studied in Ref. [29]. The effect of helicity inversion on LNV transition rates at the LHC has been studied in the context of a type- 1 seesaw model in Ref. [30]. For complementarity between $Z^{\prime}$ and $W_{R}$ searches in the LRSM, see Ref. [31]. While LHC searches are mostly sensitive to $M_{N} \sim \mathcal{O}(100) \mathrm{GeV}$-few $\mathrm{TeV}$, and $M_{W_{R}}$ up to a few $\mathrm{TeV}$, the rare LNV and LNC semileptonic meson decays on the other hand are sensitive to a much smaller $\mathrm{RH}$ neutrino mass range $M_{N} \sim(\mathrm{MeV}$-few $\mathrm{GeV})$, and to a much higher value of the $W_{R}$ gauge boson mass. These searches are thus complementary to LHC searches. It is well known that for very light and heavy neutrino masses the rates of these LNV meson and tau decays are extremely suppressed 
$[21,24]$, well below the sensitivity reach of any future experiment. This changes dramatically if there exists a heavy neutrino in the $\mathrm{MeV}-\mathrm{GeV}$ mass domain, which can be produced on shell in the parent meson decay. This can lead to large resonant enhancement of these processes. Various ongoing experiments-including NA62, LHCb, and Belle-II-are searching for the LNV meson decays. The LHCb experiment looked for the process $B^{-} \rightarrow \mu^{-} \mu^{-} \pi^{+}$, and has constrained the light neutrino-heavy neutrino mixing [15]. Due to the order-of-magnitude increase in the meson and tau flux and the long detector distance in experiments such as SHiP, MATHUSLA, and others under discussion, one expects to achieve better sensitivity for LNV meson and tau decays in the future. Even nonobservations can set tight limits on the relevant parameter space, such as on the heavy neutrino mass $M_{N}$ and RH gauge boson mass $M_{W_{R}}[21,32]$.

In this work, we study the three-body LNV and LNC meson decays $-M_{1}^{+} \rightarrow l_{i}^{+} l_{j}^{+} M_{2}^{-}$and $M_{1}^{+} \rightarrow l_{i}^{+} l_{j}^{-} M_{2}^{+}$for the LRSM, in particular focusing on the implications of possible interference effects that may occur due to degenerate or nearly degenerate RH neutrino states. With only a single heavy neutrino, the rates of LNV and LNC meson decays via RH neutrino mediation are predicted to be the same, irrespective of any $\mathrm{CP}$-violating phase present in the $\mathrm{RH}$ neutrino mixing matrix. This scenario changes dramatically if more than one heavy neutrino state contributes in these processes with a nontrivial $\mathrm{RH}$ neutrino mixing matrix. In this case, the predictions for the LNV and LNC meson decay rates can differ widely due to the interference among the contributions of different $\mathrm{RH}$ neutrinos. This leads to a change in the interpretation of data in the LRSM compared to the case of a single heavy neutrino. For a study of interference effects in semileptonic meson decays for a pure sterile neutrino without any additional gauge extension, see Ref. [25]. The $C P$ violation in semileptonic decays of charged mesons with nearly degenerate heavy neutrinos has been extensively studied in Refs. [33-36], and for $C P$ violation in rare $\tau^{ \pm}$decays with nearly equal heavy neutrino masses, see Ref. [37]. A comprehensive study of heavy neutrino oscillation in rare $W$ decays as well as tau decays with degenerate heavy neutrinos has been performed in Refs. [38-40]. The interference effect in the LRSM, relevant for collider searches, has been discussed in Ref. [41]. The interference effects have also been studied in type-I and generalized inverse seesaw models in Ref. [42]. The enhancement of $C P$ asymmetry at the LHC has been studied for heavy degenerate neutrinos in Ref. [43] and for degenerate scalars in Ref. [44].

To quantify the interference effect in meson decays, we consider $K$ - and $B$-meson LNV and LNC semileptonic three-body decays with a pion in the final state as illustrative examples. We develop the generic theory framework with two degenerate or nearly degenerate $\mathrm{RH}$ neutrino states that contribute significantly in the LNV and
LNC semileptonic three-body meson decays. Using this, we then evaluate the analytic results for the partial decay widths and branching ratios in the presence of interference terms in the amplitude. We focus on the final states that contain electrons and muons. We consider two different mass ranges of the two RH neutrino states, $0.14 \mathrm{GeV}<$ $M_{N}<0.49 \mathrm{GeV}$ and $0.14 \mathrm{GeV}<M_{N}<5 \mathrm{GeV}$, relevant for $K^{+} \rightarrow e^{+} e^{+} \pi^{-} / e^{+} \mu^{ \pm} \pi^{\mp}$ and $B^{+} \rightarrow e^{+} e^{+} \pi^{-} / e^{+} \mu^{ \pm} \pi^{\mp}$ meson decays, respectively. These decay modes, including the flavor-violating LNC modes $K^{+} \rightarrow e^{+} \mu^{-} \pi^{+}$and $B^{+} \rightarrow e^{+} \mu^{-} \pi^{+}$, are absent in the $\mathrm{SM}$, and hence serve as a clear indication of new physics.

The paper is organized as follows. In Sec. II we review the basic features of the LRSM, and in Sec. III we discuss in detail the RH neutrino contributions in LNV and LNC meson decays. In Sec. IV we discuss our results with the assumption that only two RH neutrinos are contributing with an effective $2 \times 2 \mathrm{RH}$ neutrino mixing matrix. In Sec. V we discuss the interference effects with two RH neutrinos considering the full $3 \times 3 \mathrm{RH}$ neutrino mixing matrix. Finally, we summarize our most important findings in the conclusion.

\section{LEFT-RIGHT SYMMETRIC MODEL}

The LRSM is a simple extension of the SM, where both the left and right chiral fermions are treated on an equal footing. The model is based on the gauge group $S U(3)_{c} \otimes S U(2)_{L} \otimes S U(2)_{R} \otimes U(1)_{B-L}$, where the left and right chiral fermions are doublets of $S U(2)_{L}$ and $S U(2)_{R}$, respectively. The model necessarily contains three RH neutrinos $\left(N_{R i}\right)$, which are part of the three righthanded lepton doublets. The electric charge generator $Q$ is related to the third component of weak isospins $I_{3 L}$ and $I_{3 R}$ as $Q=I_{3 L}+I_{3 R}+(B-L) / 2$. The scalar sector is also enlarged because of the extra symmetry. The LRSM contains one bidoublet $\Phi$ and two scalar triplets $\Delta_{R}$ and $\Delta_{L}$ under $S U(2)_{R}$ and $S U(2)_{L}$, respectively. The particle content is given below:

$$
\begin{gathered}
l_{L}=\left[\begin{array}{c}
\nu_{L i} \\
e_{L i}
\end{array}\right], \quad l_{R}=\left[\begin{array}{c}
N_{R i} \\
e_{R i}
\end{array}\right], \\
Q_{L}=\left[\begin{array}{c}
u_{L i} \\
d_{L i}
\end{array}\right], \quad Q_{R}=\left[\begin{array}{c}
u_{R i} \\
d_{R i}
\end{array}\right], \\
\Phi=\left[\begin{array}{cc}
\phi_{1}^{0} & \phi_{2}^{+} \\
\phi_{1}^{-} & \phi_{2}^{0}
\end{array}\right], \quad \Delta_{L / R}=\left[\begin{array}{cc}
\Delta_{L / R}^{+} / \sqrt{2} & \Delta_{L / R}^{++} \\
\Delta_{L / R}^{0} & -\Delta_{L / R}^{+} / \sqrt{2}
\end{array}\right] .
\end{gathered}
$$

The $S U(2)$ doublets $l_{L}$ and $l_{R}$ have the charges $(1,2,1,-1)$ and $(1,1,2,-1)$, while the Higgs multiplets have the charges $\Phi \sim(1,2,2,0), \quad \Delta_{L} \sim(1,3,1,+2), \quad$ and $\Delta_{R} \sim(1,1,3,+2)$. The bidoublet being neutral under $B-L$, additional Higgs triplets are required to break the 
left-right symmetric gauge group to the SM gauge group $S U(2)_{L} \otimes U(1)_{Y}$. The neutral component of $\Delta_{R}$ takes the vacuum expectation value (VEV) $v_{R}$ and breaks the gauge group $S U(2)_{R} \otimes U(1)_{B-L}$ to $U(1)_{Y}$. In the next step, the VEV of the bidoublet $\Phi$ breaks the SM gauge group to $U(1)_{Q}$. The VEV of the bidoublet is denoted as $\langle\Phi\rangle=\operatorname{Diag}\left(\frac{\kappa_{1}}{\sqrt{2}}, \frac{\kappa_{2}}{\sqrt{2}}\right)$. Due to the strong constraint on the $\rho$ parameter, the VEV of $\Delta_{L}$ should be very small, $v_{L}<$ $5 \mathrm{GeV}$ [45]. Hence, the different VEVs of $S U(2)$ triplets and the bidoublet follow the hierarchy $v_{L} \ll \kappa_{1,2} \ll v_{R}$. The Yukawa Lagrangian responsible for generating the lepton masses has the following form:

$$
\begin{aligned}
-\mathcal{L}_{Y}= & y \bar{l}_{L} \Phi l_{R}+\tilde{y} \bar{l}_{L} \tilde{\Phi} l_{R}+y_{L} l_{L}^{T} C^{-1} i \sigma^{2} \Delta_{L} l_{L} \\
& +y_{R} l_{R}^{T} C^{-1} i \sigma^{2} \Delta_{R} l_{R}+\text { H.c. },
\end{aligned}
$$

where $C$ is the charge-conjugation operator, $C=i \gamma^{2} \gamma^{0}$, and $\tilde{\Phi}=\sigma^{2} \Phi^{*} \sigma^{2}$. Here $\gamma^{\mu}$ and $\sigma^{i}$ are the Dirac and Pauli matrices, and $y, \tilde{y}, y_{L}$, and $y_{R}$ are the Yukawa couplings, respectively. After spontaneous symmetry breaking, the neutral lepton mass matrix is obtained as

$$
\mathcal{M}_{\nu}=\left[\begin{array}{ll}
M_{L} & M_{D} \\
M_{D}^{T} & M_{R}
\end{array}\right]
$$

In the above, the Dirac mass matrix $M_{D}=\frac{1}{\sqrt{2}}\left(y \kappa_{1}+\right.$ $\left.\tilde{y} \kappa_{2}\right)=y_{D} \kappa_{s}$, and $M_{L, R}$ are given by $M_{L}=\sqrt{2} v_{L} y_{L}$ and $M_{R}=\sqrt{2} v_{R} y_{R}$. The Higgs triplets $\Delta_{R}$ and $\Delta_{L}$ generate Majorana masses of heavy and light neutrinos, respectively. The parameter $\kappa_{s}$ is the electroweak VEV, and is related to $\kappa_{1,2}$ as $\kappa_{s}=\sqrt{\kappa_{1}^{2}+\kappa_{2}^{2}}$. The light and heavy neutrino masses can be calculated by using the seesaw approximation $M_{L} \ll M_{D} \ll M_{R}$. This leads to the following light and heavy neutrino mass matrix:

$$
\begin{aligned}
M_{\nu} & \sim M_{L}-M_{D} M_{R}^{-1} M_{D}^{T}+\mathcal{O}\left(M_{R}^{-2}\right) \\
& \sim \sqrt{2} v_{L} y_{L}-\frac{\kappa_{s}^{2}}{\sqrt{2} v_{R}} y_{D} y_{R}^{-1} y_{D}^{\top}
\end{aligned}
$$

and

$$
\begin{gathered}
M_{N} \sim M_{R}+\mathcal{O}\left(M_{R}^{-1}\right) \\
\sim \sqrt{2} y_{R} v_{R} .
\end{gathered}
$$

The mass matrix $\mathcal{M}_{\nu}$ in Eq. (5) can be diagonalized by a unitary transformation,

$$
\mathcal{V}^{T}\left[\begin{array}{ll}
M_{L} & M_{D} \\
M_{D}^{T} & M_{R}
\end{array}\right] \mathcal{V}=\left[\begin{array}{cc}
\tilde{\mathcal{M}}_{\nu} & 0 \\
0 & \tilde{\mathcal{M}}_{R}
\end{array}\right],
$$

where $\quad \tilde{\mathcal{M}}_{\nu}=\operatorname{Diag}\left(m_{1}, m_{2}, m_{3}\right) \quad$ and $\quad \tilde{\mathcal{M}}_{R}=\operatorname{Diag}\left(M_{1}\right.$, $\left.M_{2}, M_{3}\right)$. Up to $\mathcal{O}\left(M_{R}^{-2}\right)$, the mixing matrix $\mathcal{V}$ has the following form:

$$
\mathcal{V}=\left[\begin{array}{cc}
U_{\mathrm{PMNS}} & S^{\dagger} \\
T & K_{R}^{\dagger}
\end{array}\right],
$$

where $S, T \approx M_{D} M_{R}^{-1}$. In the above, $K_{R}$ is the diagonalization matrix for the RH neutrino mass matrix $M_{R}$. We will neglect the effect of $S, T$ in our subsequent discussions, as $S, T \sim \mathcal{O}\left(10^{-5}\right)$ for $M_{\nu} \sim \mathcal{O}(0.1) \mathrm{eV}$ and $M_{N} \sim \mathcal{O}(1 \mathrm{GeV})$.

\section{A. Gauge sector}

In addition to the SM gauge bosons $W_{L}$ and $Z$, this model also has a RH gauge boson $W_{R}$ and an additional neutral gauge boson $Z^{\prime}$. The left- and right-handed charged gauge bosons $\left(W_{L}, W_{R}\right)$ will mix and the mixing angle can be approximated to be

$$
\zeta \simeq \frac{\kappa_{1} \kappa_{2}}{v_{R}^{2}} \simeq 2 \frac{\kappa_{2}}{\kappa_{1}}\left(\frac{M_{W_{L}}}{M_{W_{R}}}\right)^{2} .
$$

Due to this small mixing between the charged gauge bosons, the masses of the gauge bosons can be approximated as

$$
M_{W_{L}} \simeq M_{W_{1}} \simeq \frac{g \kappa_{1}}{\sqrt{2}}, \quad M_{W_{R}} \simeq M_{W_{2}} \simeq g v_{R}
$$

Note that throughout our calculation we assume $g \equiv g_{L}=g_{R}$, which is justified as we consider parity as a symmetry in the LRSM. The mass of the neutral gauge boson $Z^{\prime}$ for this choice becomes $M_{Z^{\prime}} \sim 1.7 M_{W_{R}}$.

\section{B. Charged- and neutral-current Lagrangian}

The charged-current Lagrangian for the quark sector has the following form:

$$
\begin{aligned}
\mathcal{L}_{c c}^{q}= & \frac{g}{\sqrt{2}} \sum_{i, j} \bar{u}_{i} V_{i j}^{\mathrm{CKM}} W_{L \mu}^{+} \gamma^{\mu} P_{L} d_{j} \\
& +\frac{g}{\sqrt{2}} \sum_{i, j} \bar{u}_{i} V_{i j}^{\mathrm{R}-\mathrm{CKM}} W_{R \mu}^{+} \gamma^{\mu} P_{R} d_{j}+\text { H.c. },
\end{aligned}
$$

where $P_{L}=\frac{1}{2}\left(1-\gamma_{5}\right)$ and $P_{R}=\frac{1}{2}\left(1+\gamma_{5}\right)$. In our analysis, we consider $V^{\mathrm{R}-\mathrm{CKM}}$ to be the same as $V^{\mathrm{CKM}}$. This holds naturally if parity is realized as a symmetry in the LRSM together with the phase of the bidoublet Higgs VEV considered to be zero $[46,47]$. The charged-current Lagrangian for the lepton-neutrino sector is given by 


$$
\begin{aligned}
\mathcal{L}_{C C}^{l} & =\frac{g}{\sqrt{2}} \sum_{i} \bar{l}_{L_{i}} W_{L \mu}^{-} \gamma^{\mu} P_{L}\left(U_{\mathrm{PMNS}} \nu_{L}+S^{\dagger} N^{c}\right)_{i} \\
& +\frac{g}{\sqrt{2}} \sum_{i} \bar{l}_{R_{i}} W_{R \mu}^{-} \gamma^{\mu} P_{R}\left(K_{R}^{T} N+T^{*} \nu_{L}^{c}\right)_{i}+\text { H.c. }
\end{aligned}
$$

Note that in a few of the decay channels of $N_{i}$, the neutral current will also contribute. The neutral current for the LRSM has the following form $[48,49]$ :

$$
\mathcal{L}_{\mathrm{NC}}=\frac{g}{\cos \theta_{w}}\left(Z_{\mu} J_{Z}^{\mu}+\frac{\cos ^{2} \theta_{w}}{\sqrt{\cos 2 \theta_{w}}} Z_{\mu}^{\prime} J_{Z^{\prime}}^{\mu}\right),
$$

where

$$
\begin{gathered}
J_{Z}^{\mu}=\sum_{i} \bar{f}_{i} \gamma^{\mu}\left(I_{3 L} P_{L}-Q \sin ^{2} \theta_{w}\right) f_{i}, \\
J_{Z^{\prime}}^{\mu}=\sum_{i} \bar{f}_{i} \gamma^{\mu}\left(I_{3 R} P_{R}-\tan ^{2} \theta_{w}\left(Q-I_{3 L}\right)\right) f_{i} .
\end{gathered}
$$

As emphasized before, for the particular choice of the neutrino mixing matrix $\mathcal{V}$, we neglect interaction terms proportional to the mixing matrices $S$ and $T$.

Note that the masses of both the RH gauge bosons and $\mathrm{RH}$ neutrinos are proportional to the $S U(2)_{R}$ breaking scale $v_{R}$. However, since the RH neutrino masses also depend on the Yukawa couplings of $\Delta_{R}$ with the heavy neutrinos, one can choose to have a wide splitting between the two. In this paper we consider the masses of the heavy neutrino in the $\mathrm{MeV}_{-}$ $\mathrm{GeV}$ range, in particular, in between $0.14 \mathrm{GeV}<M_{N_{i}}<$ $5 \mathrm{GeV}$, so that the decay of the mesons can produce on-shell RH neutrinos. Semileptonic meson decays such as $M_{1}^{+} \rightarrow$ $l_{i}^{+} l_{j}^{ \pm} M_{2}^{\mp}$ will then be resonantly enhanced due to the on-shell production of the RH neutrinos.

\section{LNV AND LNC MESON DECAYS}

Lepton number is broken in the LRSM due to $S U(2)_{R} \times$ $U(1)_{B-L}$ symmetry breaking. The heavy neutrinos being Majorana particles can result in LNV as well as LNC meson decay processes:

$$
\begin{aligned}
& \mathrm{LNV}: M_{1}^{+}(p) \rightarrow l_{i}^{+}\left(k_{1}\right)+l_{j}^{+}\left(k_{2}\right)+M_{2}^{-}\left(k_{3}\right), \\
& \mathrm{LNC}: M_{1}^{+}(p) \rightarrow l_{i}^{+}\left(k_{1}\right)+l_{j}^{-}\left(k_{2}\right)+M_{2}^{+}\left(k_{3}\right) .
\end{aligned}
$$

In the above, $M_{1}$ is a pseudoscalar meson and $M_{2}$ can be either a pseudoscalar or a vector meson. Here we consider only the case of a pseudoscalar meson $M_{2}$. The LNV mode for all flavors of final-state leptons arises entirely from RH neutrino mediation. Also, for the LNC mode with different lepton flavors (such as a $e^{+} \mu^{-}$combination) the contribution arises solely from RH neutrino mediation. These processes are absent in the SM, and hence serve as a distinct signature of new physics. On the other hand, for the
LNC mode with the same lepton flavors $\left(e^{+} e^{-}, \mu^{+} \mu^{-}\right)$, virtual photon and virtual $Z$ diagrams (one-loop penguin diagrams) will also contribute in addition to the $\mathrm{RH}$ neutrino contribution with a substantial branching ratio $\sim 10^{-7} / 10^{-8}$ for $K^{+} \rightarrow e^{+} e^{-} \pi^{-} / \mu^{+} \mu^{-} \pi^{+}$[50-52]. In our subsequent discussions, the contribution to such LNC processes coming from diagrams involving $\mathrm{RH}$ neutrino mediation will be referred to as the new physics (NP) contribution. In the rest of the paper, we focus mainly on the RH neutrino contribution to the LNV and LNC meson decays, as the main focus of this paper is on the interference effect of RH neutrino states.

For our later discussion on the RH neutrino contribution in LNV and LNC semileptonic meson decays, which is the main subject matter of this paper, we assume that there are at least two RH neutrinos with masses in the range $100 \mathrm{MeV}-5 \mathrm{GeV}$ mediating these meson decays. The Feynman diagrams for the LNV process are shown in Fig. 1. The different contributions are mediated through $W_{L}-N_{k}-W_{L}, \quad W_{L}-N_{k}-W_{R}, \quad W_{R}-N_{k}-W_{L}, \quad$ and $W_{R}-N_{k}-W_{R}$, respectively. Note that, while the $W_{R}-$ $N_{k}-W_{R}$ diagram completely depends on the mixing matrix in the RH neutrino sector, the other diagrams also depend on the light-heavy neutrino mixing. Throughout this work, we consider the contribution from the $W_{R}-$ $N_{k}-W_{R}$ diagram only, as the light-heavy neutrino mixing angle that comes from the off-diagonal blocks $(S, T)$ of the mixing matrix $\mathcal{V}$ is very small. Considering the heavy neutrinos to be $\mathcal{O}(\mathrm{MeV})$, the $\mathrm{RH}$ neutrinos can be produced on shell and the semileptonic meson decay will be resonantly enhanced. In addition, there can also be contributions from $W_{L}-W_{R}$ mixing in one of the legs, but these are suppressed due to the small mixing angle $\zeta$. The contributions from the light-neutrino-mediated process will be much smaller due to mass suppression. Hence, we do not consider all of these other contributions in our analysis. In Fig. 2 we show the Feynman diagram for the LNC process.

The contribution from heavy neutrinos $N_{a}$ to the decay amplitude of the LNV process $M_{1}^{+}(p) \rightarrow l_{i}^{+}\left(k_{1}\right) l_{j}^{+}\left(k_{2}\right)$ $M_{2}^{-}\left(k_{3}\right)$ can be written as

$$
\mathcal{M}_{i j}^{\mathrm{LNV}, a}=\left(\mathcal{M}_{\mathrm{lep}}^{\mu \nu}\right)_{i j}^{a} \mathcal{M}_{\mu \nu}^{\mathrm{had}},
$$

where

$$
\begin{aligned}
\mathcal{M}_{\mu \nu}^{\mathrm{had}}= & \frac{G_{F}}{\sqrt{2}} M_{W_{L}}^{2} V_{M_{1}}^{\mathrm{CKM}} V_{M_{2}}^{\mathrm{CKM}} \\
& \times\left\langle 0\left|\bar{q}_{2} \gamma_{\mu} \gamma^{5} q_{1}\right| M_{1}^{+}(p)\right\rangle\left\langle M_{2}^{+}\left(k_{3}\right)\left|\bar{q}_{3} \gamma_{\nu} \gamma^{5}\right| 0\right\rangle \\
= & \frac{G_{F}}{\sqrt{2}} M_{W_{L}}^{2} V_{M_{1}}^{\mathrm{CKM}} V_{M_{2}}^{\mathrm{CKM}} f_{M_{1}} f_{M_{2}} p_{\mu} k_{3 \nu} .
\end{aligned}
$$

In the above, $G_{F}$ is the Fermi coupling constant, $V_{M_{1}}^{\mathrm{CKM}}$ $\left(V_{M_{2}}^{\mathrm{CKM}}\right)$ are the Cabibbo-Kobayashi-Maskawa (CKM) 

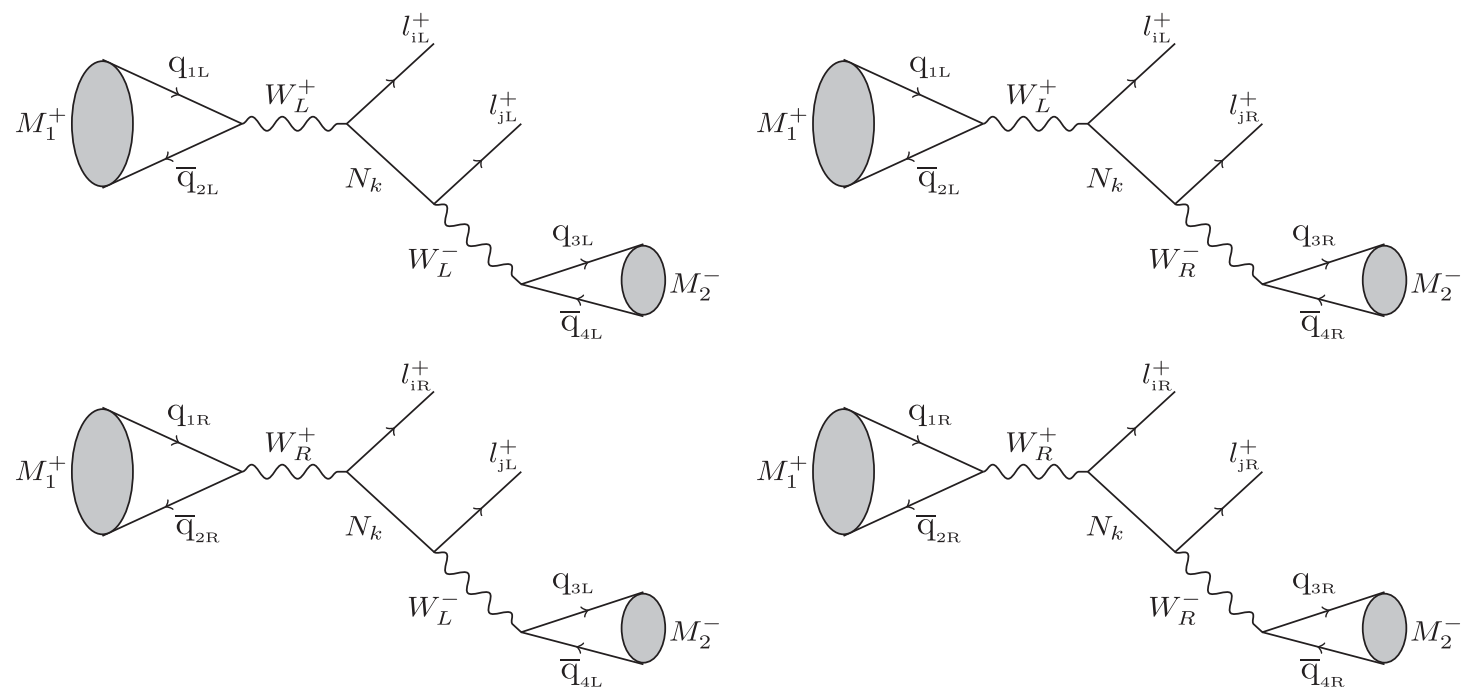

FIG. 1. Feynman diagrams for LNV meson decays. See text for details.

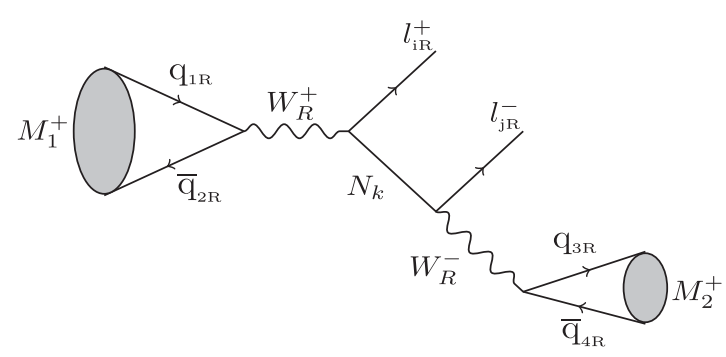

FIG. 2. Feynman diagram for LNC meson decay, mediated via the RH neutrino.

matrix elements at the decay (production) vertex of the meson $M_{1}\left(M_{2}\right)$, and $f_{M_{1}}, f_{M_{2}}$ are the decay constants of $M_{1}, M_{2}$. The relevant leptonic matrix element for the $(\Delta L=2) \mathrm{LNV}$ process is given by the following product of two charged currents:

$$
\begin{aligned}
\mathcal{M}_{\text {lep }}^{\mu \nu} & \propto\left(\bar{N} \gamma^{\mu} P_{R} l\right)\left(\bar{N} \gamma^{\nu} P_{R} l\right) \\
& =\left(\bar{N}_{a}\left(K_{R}^{*}\right)_{a i} \gamma^{\mu} P_{R} l_{i}\right)\left(\bar{N}_{a}\left(K_{R}^{*}\right)_{a j} \gamma^{\nu} P_{R} l_{j}\right) \\
& =\left(\bar{l}_{i}^{c} K_{R a i}^{*} \gamma^{\mu} P_{L} N_{a}\right)\left(\bar{N}_{a} K_{R a j}^{*} \gamma^{\nu} P_{R} l_{j}\right),
\end{aligned}
$$

where we have used the fact that massive neutrinos are Majorana type $\left(N_{a}^{c}=N_{a}\right)$. We can now write the leptonic part of the amplitude as

$$
\begin{aligned}
\left(\mathcal{M}_{\text {lep }}^{\mu \nu}\right)_{i j}^{a}= & 2 \sqrt{2} G_{F} \frac{M_{W_{L}}^{2}}{M_{W_{R}}^{4}}\left(K_{R}^{*}\right)_{a i}\left(K_{R}^{*}\right)_{a j} \\
& \times M_{N_{a}} \frac{\bar{u}\left(k_{1}\right) \not p \mid k_{3} P_{R} v\left(k_{2}\right)}{q^{2}-M_{N_{a}}^{2}+i \Gamma_{N_{a}} M_{N_{a}}},
\end{aligned}
$$

where $q=p-k_{1}$. The $1 / M_{W_{R}}^{4}$ term appears due to the two $W_{R}$ gauge boson propagators in the bottom most panel of
Fig. 1. Finally, we can write the individual contributions from heavy neutrinos $N_{a}$ to the amplitude as

$$
\begin{aligned}
\mathcal{M}_{i j}^{\mathrm{LNV}, a}= & 2 G_{F}^{2} V_{M_{1}}^{\mathrm{CKM}} V_{M_{2}}^{\mathrm{CKM}} f_{M_{1}} f_{M_{2}}\left(\frac{M_{W_{L}}}{M_{W_{R}}}\right)^{4}\left(K_{R}^{*}\right)_{a i}\left(K_{R}^{*}\right)_{a j} \\
& \times M_{N_{a}} \frac{\bar{u}\left(k_{1}\right) \not \not k_{3} P_{R} v\left(k_{2}\right)}{q^{2}-M_{N a}^{2}+i \Gamma_{N a} M_{N a}}
\end{aligned}
$$

where $\Gamma_{N_{a}}$ is the decay width of heavy neutrinos $N_{a}$, obtained by summing over all accessible final states. Adding the contributions from all heavy neutrinos, we can write the full amplitude as

$$
\mathcal{M}^{\mathrm{LNV}}=\sum_{a=1}^{3}\left(\mathcal{M}_{i j}^{\mathrm{LNV}, a}+\mathcal{M}_{j i}^{\mathrm{LNV}, a}\right),
$$

where the second contribution comes from the exchange of two leptons. Finally, the total amplitude squared $\left|\mathcal{M}^{\mathrm{LNV}}\right|^{2}$ can be written as

$$
\begin{aligned}
\left|\mathcal{M}^{\mathrm{LNV}}\right|^{2}= & \sum_{a, b=1 ; b>a}^{3}\left(\left|\mathcal{M}_{i j}^{\mathrm{LNV}, a}\right|^{2}+\left|\mathcal{M}_{j i}^{\mathrm{LNV}, a}\right|^{2}\right. \\
& +2 \operatorname{Re}\left[\left(\mathcal{M}_{i j}^{\mathrm{LNV}, a}\right)^{\dagger}\left(\mathcal{M}_{i j}^{\mathrm{LNV}, b}\right)\right] \\
& \left.+2 \operatorname{Re}\left[\left(\mathcal{M}_{j i}^{\mathrm{LNV}, a}\right)^{\dagger}\left(\mathcal{M}_{j i}^{\mathrm{LNV}, b}\right)\right]\right) .
\end{aligned}
$$

The explicit forms of these squared matrix elements are provided in the Appendix A.

\section{A. Decay widths of RH neutrino}

The RH neutrino state $N_{i}$ of mass $\mathrm{MeV}$ to few $\mathrm{GeV}$ can decay to various final states, such as $l^{ \pm} V^{\mp}, l^{ \pm} P^{\mp}$, 

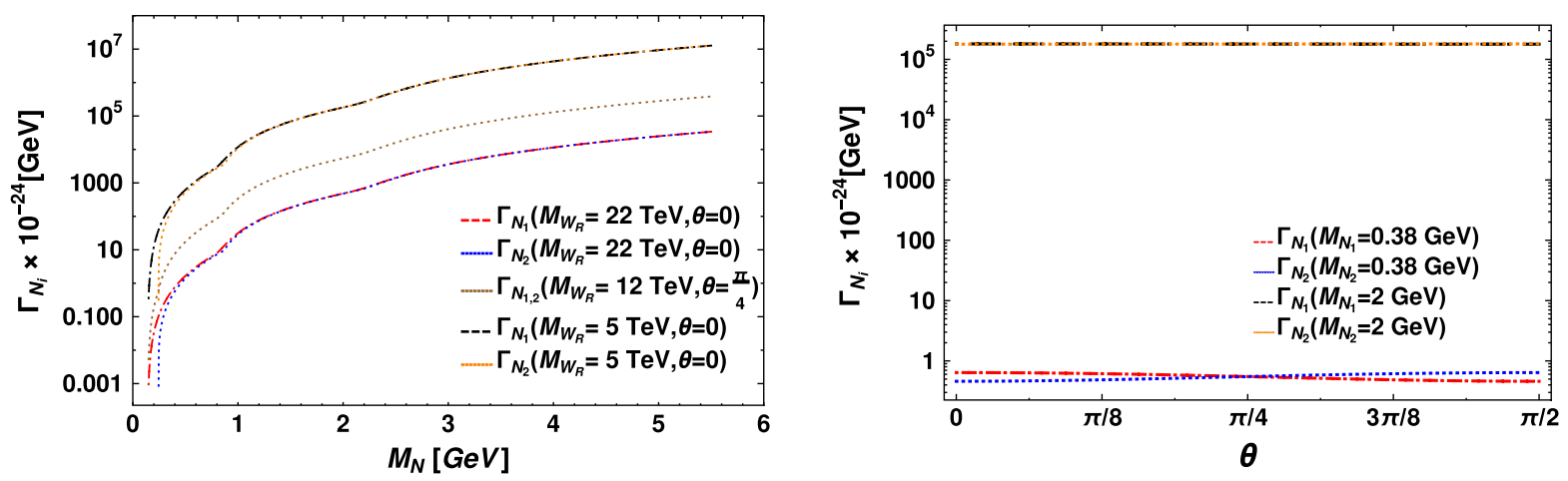

FIG. 3. Left: variation of the decay widths of the RH neutrino states $N_{1,2}$ with the masses of RH neutrinos for different values of $\theta$ and RH gauge boson mass $M_{W_{R}}$. Right: variation of the decay widths of $N_{1,2}$ with $\theta$. For $M_{N_{1,2}}=0.38(2) \mathrm{GeV}$, we consider $M_{W_{R}}=22(5) \mathrm{TeV}$, respectively.

$\nu_{l} V^{0}, \nu_{l} P^{0}$, where $V, P$ are the vector and pseudoscalar mesons, respectively. The choice of $K_{R}$ plays a crucial role in determining the different possible decay modes of the RH neutrino. Following the parametrization given in Eq. (32), which depends on only one angle and one phase, we show the decay width of the RH neutrino state $N_{1,2}$ in Fig. 3. The left panel represents the variation of the decay widths of $N_{1,2}$ with the masses of the RH neutrinos, and the right panel represents the variation with respect to the mixing angle $\theta$, where $\theta$ parametrizes the mixing between $N_{1}$ and $N_{2}$ in the two-generation case. For a detailed discussion, see Eq. (32). We find that for smaller values of $M_{N_{1,2}}$ the dependency of the decay width on mixing angles is more prominent, whereas for higher values of $M_{N_{1,2}}$ the $\theta$ dependency is negligible. This is clearly evident in the right panel, where for $M_{N}=2 \mathrm{GeV}$ both of the decay widths $\Gamma_{N_{12}}$ coincide. In the left panel, which represents decay widths for various $\theta$, the decay widths of $N_{1,2}$ show some difference for smaller masses and for $\theta \neq \pi / 4$, while for larger masses $M_{N_{1,2}}>$ $1.5 \mathrm{GeV}$ both of the decay widths are same. This will have an impact on the estimated branching ratios for $K$ and $B$ mesons, which we will discuss in the subsequent sections. From Fig. 3, it is clear that the decay width of the RH neutrino is indeed very small for our chosen mass range. Hence, we can safely use the narrow-width approximation,

$$
\begin{aligned}
\left|\mathcal{M}_{i j}^{\mathrm{LNV}, a}\right|^{2} & \propto \frac{1}{\left(\left(p-k_{1}\right)^{2}-M_{N_{a}}^{2}\right)^{2}+\Gamma_{N_{a}}^{2} M_{N_{a}}^{2}} \\
& =\frac{\pi}{M_{N_{a}} \Gamma_{N_{a}}} \delta\left(\left(p-k_{1}\right)^{2}-M_{N_{a}}^{2}\right) .
\end{aligned}
$$

We have verified that for the parametrization given in Eq. (52) the narrow-width approximation is valid too. Note that we are neglecting terms like $\left(\mathcal{M}_{i j}^{\mathrm{LNV}, a}\right)^{\dagger} \mathcal{M}_{j i}^{\mathrm{LNV}, b}$ as

$$
\begin{aligned}
\left(\mathcal{M}_{i j}^{\mathrm{LNV}, a}\right)^{\dagger} \mathcal{M}_{j i}^{\mathrm{LNV}, b} & \propto \frac{1}{\left(\left(p-k_{1}\right)^{2}-M_{N_{a}}^{2}-i \Gamma_{N_{a}} M_{N_{a}}\right)\left(\left(p-k_{2}\right)^{2}-M_{N_{b}}^{2}+i \Gamma_{N_{b}} M_{N_{b}}\right)} \\
& \neq \frac{\pi}{M_{N_{a} \Gamma_{N_{a}}}} \delta\left(\left(p-k_{1}\right)^{2}-M_{N_{a}}^{2}\right) .
\end{aligned}
$$

Hence, contributions from these terms will not be resonantly enhanced and can be safely neglected compared to other terms; see Ref. [24]. Finally, the LNV decay rate can be written as

$$
d \Gamma^{\mathrm{LNV}}=\left(1-\frac{\delta_{i j}}{2}\right) \frac{1}{2 m}\left|\mathcal{M}^{\mathrm{LNV}}\right|^{2} d_{3}\left(\mathrm{PS} ; p \rightarrow k_{1} k_{2} k_{3}\right),
$$

where $d_{3}\left(\mathrm{PS} ; p \rightarrow k_{1} k_{2} k_{3}\right)$ is the three-body phase space which can be written in terms of the product of two twobody phase spaces as follows:

$$
\begin{aligned}
& d_{3}\left(\mathrm{PS} ; p \rightarrow k_{1} k_{2} k_{3}\right) \\
& =d_{2}\left(\mathrm{PS} ; p \rightarrow k_{1} q\right) d q^{2} d_{2}\left(\mathrm{PS} ; q \rightarrow k_{2} k_{3}\right) .
\end{aligned}
$$

The full analytical expressions for the LNV decay width and three-body phase space are given in Appendix A. Similarly, the LNC process can also be mediated by the heavy neutrinos and the relevant leptonic part of the matrix element: 


$$
\begin{aligned}
& \mathcal{M}_{\text {lep }}^{\mu \nu} \propto\left(\bar{l} \gamma^{\mu} P_{R} N\right)\left(\bar{N} \gamma^{\nu} P_{R} l\right) \\
& =\left(\bar{l}_{i} \gamma^{\mu} P_{R}\left(K_{R}^{T}\right)_{i a} N_{a}\right)\left(\bar{N}_{a}\left(K_{R}^{*}\right)_{a j} \gamma^{\nu} P_{R} l_{j}\right) .
\end{aligned}
$$

The amplitude coming from individual contributions of heavy neutrinos $N_{a}$ is given by

$$
\begin{aligned}
\mathcal{M}_{i j}^{\mathrm{LNC}, a}= & 2 G_{F}^{2} V_{M_{1}}^{\mathrm{CKM}} V_{M_{2}}^{\mathrm{CKM}} f_{M_{1}} f_{M_{2}}\left(\frac{M_{W_{L}}}{M_{W_{R}}}\right)^{4}\left(K_{R}\right)_{a i}\left(K_{R}^{*}\right)_{a j} \\
& \times \frac{\bar{u}\left(k_{1}\right) \not p \phi k_{3} P_{R} v\left(k_{2}\right)}{q^{2}-M_{N_{a}}^{2}+i \Gamma_{N_{a}} M_{N_{a}}} .
\end{aligned}
$$

Following the same approach as in the LNV case, one can also derive the partial decay width for the LNC process. The details are given in Appendix A. In the subsequent sections, we will consider a simplified scenario where both RH neutrino states $N_{1,2}$ can give resonantly enhanced contributions in the LNV and LNC processes, and we will quantify the effect of the interference.

Before presenting our analysis on the interference effect, we make a few remarks about the bound appearing on such $\mathrm{RH}$ neutrino states from cosmology. Note that RH neutrinos with masses in the range $\mathrm{MeV}-\mathrm{O}(\mathrm{GeV})$ can also be constrained from cosmological considerations. A RH neutrino with a mixing angle $\theta$ with the active neutrino can decay to leptonic and hadronic final states. If the decay happens around the time of big bang nucleosynthesis (BBN) $\tau \geq 1 \mathrm{sec}$, this can alter the prediction for the light element abundance in the Universe. Constraints from BBN on the $\mathrm{MeV}$-scale $\mathrm{RH}$ neutrino have been discussed in detail in Ref. [53], with the assumption of a pure sterile neutrino. See also Refs. [54,55] for a generic discussion on BBN constraints for a late-decaying massive state $X$. Similar constraints would also be applicable for the LRSM, where the RH neutrino decays via off-shell $W_{R}$ gauge bosons and leads to semileptonic final states. We estimate the lifetime of the RH neutrino states for $380 \mathrm{MeV}$ and $2 \mathrm{GeV}$, and the RH gauge boson masses as $22 \mathrm{TeV}$ and $5 \mathrm{TeV}$, respectively. We find that the RH neutrino lifetime varies between $10^{-6}-1 \mathrm{sec}$. For these RH neutrino masses, a $W_{R}$ gauge boson heavier than $22 \mathrm{TeV}$ will be tightly constrained from BBN. Note that the decay width/lifetime of the RH neutrino states can be made larger/smaller if additional channels for RH neutrino decay open up. For example, with a sizable active-sterile mixing $S, T$ in Eq. (13), the decay lifetime can be made sufficiently smaller, and hence BBN constraints can be avoided. In that scenario, additional diagrams $L-R, L-L$ shown in Fig. 1 will also contribute in three-body LNV and LNC meson decays. A detailed evaluation of these processes is beyond the scope of this paper, and will be pursued elsewhere.

\section{ANALYSIS FOR THE CASE OF TWO GENERATIONS}

We consider the case where two of the heavy neutrino states are degenerate or nearly degenerate, i.e., $M_{N_{1}} \sim M_{N_{2}}$ and $M_{N_{3}}$ is very heavy. The two degenerate states mediate the LNV and LNC meson decays of $K$ and $B$ mesons, and give resonance enhancement in the branching ratios, being in the $\mathrm{MeV}-\mathrm{GeV}$ mass domain. The choice of the $\mathrm{RH}$ neutrino masses can be justified, as the free parameters $y_{D}$ and $y_{R}$ in the Lagrangian (4) that can be adjusted to get $\mathrm{eV}$ light neutrino mass, along with two nearly degenerate $\mathrm{RH}$ neutrinos. The matrix $K_{R}$ is in general a $3 \times 3$ unitary matrix with few angles and phases. However, to present the effect of interference in a more simplified way, we choose the following parametrization of $K_{R}$ :

$$
K_{R}=\left[\begin{array}{ccc}
\cos \theta & -\sin \theta & 0 \\
e^{-i \phi} \sin \theta & e^{-i \phi} \cos \theta & 0 \\
0 & 0 & 1
\end{array}\right] .
$$

The above matrix $K_{R}$ is just the product of an orthogonal matrix with angle $\theta$ and a diagonal phase matrix. By choosing such a parametrization, we are interested only in mixing between the two flavors $\left(N_{1}, N_{2}\right)$ which are assumed to have degenerate or nearly degenerate masses. The parametrization of the mixing matrix $K_{R}$ enables the three-body decay of the meson into $e$ and $\mu$ lepton flavors, and suppresses any final state with a tau. In Secs. IV A and IV B we explicitly demonstrate the impact of this angle and phase on LNV and LNC meson decay rates.

Note that in addition to the mixing angle and phase, the contributions of $N_{1}$ and $N_{2}$ states in LNV and LNC decays also depend on the mass difference of the RH neutrino states $N_{1,2}$, along with their decay widths $\Gamma_{N_{1,2}}$. For degenerate or nearly degenerate masses of $\mathrm{RH}$ neutrinos, the states $N_{1,2}$ will both be resonantly produced in the $K, B$ meson decays. Depending on the angle and phases of the mixing matrix, the contributions of the $N_{1,2}$ states can interfere either constructively or destructively. For a very large mass difference between the $N_{1,2}$ states, the interference effect will fade away. Therefore, for a large mass splitting between the RH neutrinos, the LNV and LNC meson decays are similar to the one-generation case, which has been studied in detail in Refs. [14,19,21,24]. To demonstrate the effect of the mass splitting $\delta M$ and the decay width $\Gamma=\frac{\Gamma_{N_{1}}+\Gamma_{N_{2}}}{2}$ on the interference effect, in Fig. 4 we show the ratio of LNV and LNC branching ratios for different values of the $\delta M / \Gamma$, where we consider the $K^{+} \rightarrow$ $e^{+} e^{ \pm} \pi^{\mp}$ channel as an illustrative example. The left panel shows the new physics contribution, mediated via RH neutrinos (referred to as NP in the plot). In the right panel we show a conservative estimate of the LNV and LNC ratio. The ratio of the mass difference between the two states $N_{1,2}$ 

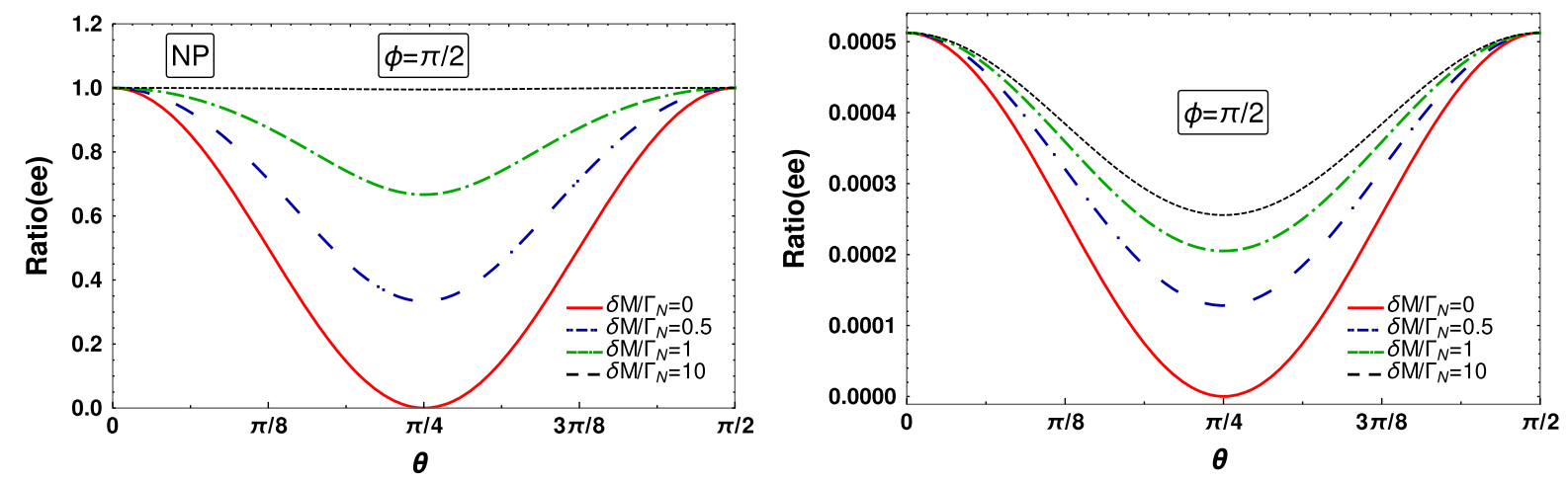

FIG. 4. Variation of the ratio of LNV and LNC branching ratios of $K^{+} \rightarrow e^{+} e^{ \pm} \pi^{\mp}$ with the mixing angle $\theta$. The red solid, blue dashdouble-dotted, green dash-dotted, and black dashed lines represent four different $\delta M / \Gamma$ ratios $(0,0.5,1,10)$. The RH neutrino mass has been set at $M_{N} \sim 0.38 \mathrm{GeV}$. The left panel represents only the RH-neutrino-mediated contribution. In the right panel we present a conservative estimate by including the "polluting" SM contribution maximally.

and the decay width $\Gamma$ has a large impact on the interference effect. Without any interference effect, the LNV and LNC decay branching ratios would be the same, leading the ratio to be identity. For a very small value of $\delta M / \Gamma$, the ratio deviates significantly from unity. As is evident from the figure, by increasing the $\delta M / \Gamma$ ratio the interference between $N_{1,2}$ state tends to become less prominent, but still has a visible effect for $\delta M \sim \Gamma$. For much larger values, such as $\delta M / \Gamma=10$, the interference effect and the oscillatory behavior fade away, causing the LNV and LNC branching ratios to be equal. While we present the discussion on the ee final state, it is to be noted that this is not the most optimal channel due to large SM contributions in the LNC process. However, the left panel of Fig. 4 can be used to demonstrate the dependency of the interference effect on $\delta M / \Gamma_{N}$. We have verified that this feature is present in the $\mu \mu$ and $e \mu$ channels (for nondegenerate RH neutrino masses) with the $K_{R}$ matrix given in Eq. (32), and also in the $e \mu$ channel for the parametrization of $K_{R}$ discussed in Sec. V.

\section{A. LNV}

We give the complete list of decay channels of $N_{i}$ for a general light-heavy mixing matrix in Appendix C. We list only the channels that are kinematically allowed for RH neutrino masses in the range $0.14 \mathrm{GeV}<M_{N_{i}}<5 \mathrm{GeV}$. Note that for our chosen mass range of RH neutrino states and with the off-diagonal mixing elements $S, T \sim 0$, decay modes such as $N \rightarrow l^{ \pm} P^{\mp}, l^{\mp} V^{ \pm}, \nu_{l} P^{0}$, and $\nu_{l} V^{0}$ will only be allowed. With the mixing matrix $K_{R}$ given in Eq. (32), the RH neutrino decay width will also depend on the mixing angle $\theta$. One can write down a generic expression for the total decay width of degenerate $\mathrm{RH}$ neutrinos as

$$
\Gamma_{N_{1}}=\frac{1}{M_{W_{R}}^{4}}\left(A\left(M_{N}\right)+B\left(M_{N}\right) \cos 2 \theta\right),
$$

$$
\Gamma_{N_{2}}=\frac{1}{M_{W_{R}}^{4}}\left(A\left(M_{N}\right)-B\left(M_{N}\right) \cos 2 \theta\right) .
$$

Note that the above expressions can be obtained by substituting $K_{R}$ into the expressions of the decay widths given in Appendix $\mathrm{C}$, where we consider $S, T \sim 0$. For the choice of $M_{N_{1}} \sim M_{N_{2}} \sim M_{N}=0.38 \mathrm{GeV}$, which we consider for $K^{+}$meson decay, the RH neutrinos will only have decay modes with a charged pion in the final state, i.e., $N_{j} \rightarrow l_{i}^{ \pm}+\pi^{\mp}$. The expressions of the two functions $A\left(M_{N}\right)$ and $B\left(M_{N}\right)$ have a simpler form, and are given in Appendix C [see Eqs. (C10) and (C11)]. We note that for a relatively larger mass $M_{N_{i}}>1.5 \mathrm{GeV}$ of the $\mathrm{RH}$ neutrino states, the decay widths are nearly independent of the mixing angle $\theta$ (Fig. 3). This can be understood easily as follows. We can neglect the final-state lepton masses in evaluating the decay widths for RH neutrinos with masses $M_{N} \sim$ few $\mathrm{GeV}$. The unitarity relation for the mixing matrix $K_{R}$ makes the total decay widths of $N_{1,2}$ nearly independent of $\theta$. In this case, one can write down the expression for the total decay width as

$$
\Gamma_{N_{1}} \sim \frac{1}{M_{W_{R}}^{4}} A\left(M_{N_{1}}\right), \quad \Gamma_{N_{2}} \sim \frac{1}{M_{W_{R}}^{4}} A\left(M_{N_{2}}\right) .
$$

Here $A\left(M_{N}\right)$ is a function of the mass $M_{N}$ and can be derived from different decay modes, given in Appendix C. Therefore, for degenerate masses $\left(M_{N_{1}}=M_{N_{2}} \equiv M_{N}\right)$, the above two decay widths will be nearly the same.

Using the general form of the heavy $N$ decay width given in Eqs. (33) and (34), one can write down the LNV decay rate of the parent meson $M_{1}$ in terms of same-flavor finalstate leptons, which leads to a rather complicated expression. The explicit expressions for the $e e$ and $\mu \mu$ cases are as follows: 


$$
\begin{aligned}
\Gamma_{e e}^{\mathrm{LNV}}= & \pi \beta M_{N}\left(\frac{\cos ^{4} \theta}{A\left(M_{N}\right)+B\left(M_{N}\right) \cos 2 \theta}+\frac{\sin ^{4} \theta}{A\left(M_{N}\right)-B\left(M_{N}\right) \cos 2 \theta}\right. \\
& \left.+\frac{1}{4} \cos 2 \phi \sin ^{2} 2 \theta\left[\frac{1}{A\left(M_{N}\right)+B\left(M_{N}\right) \cos 2 \theta}+\frac{1}{A\left(M_{N}\right)-B\left(M_{N}\right) \cos 2 \theta}\right]\right) f^{e e}\left(M_{N}\right), \\
\Gamma_{\mu \mu}^{\mathrm{LNV}}= & \pi \beta M_{N}\left(\frac{\sin ^{4} \theta}{A\left(M_{N}\right)+B\left(M_{N}\right) \cos 2 \theta}+\frac{\cos ^{4} \theta}{A\left(M_{N}\right)-B\left(M_{N}\right) \cos 2 \theta}\right. \\
& \left.+\frac{1}{4} \cos 2 \phi \sin ^{2} 2 \theta\left[\frac{1}{A\left(M_{N}\right)+B\left(M_{N}\right) \cos 2 \theta}+\frac{1}{A\left(M_{N}\right)-B\left(M_{N}\right) \cos 2 \theta}\right]\right) f^{\mu \mu}\left(M_{N}\right) .
\end{aligned}
$$

The partial decay width for different-flavor final-state leptons is given by

$$
\begin{aligned}
\Gamma_{e \mu}^{\mathrm{LNV}}= & \pi \beta M_{N}\left(\frac{\sin ^{2} 2 \theta}{4\left(A\left(M_{N}\right)+B\left(M_{N}\right) \cos 2 \theta\right)}+\frac{\sin ^{2} 2 \theta}{4\left(A\left(M_{N}\right)-B\left(M_{N}\right) \cos 2 \theta\right)}\right. \\
& \left.-\frac{1}{4} \cos 2 \phi \sin ^{2} 2 \theta\left[\frac{1}{A\left(M_{N}\right)+B\left(M_{N}\right) \cos 2 \theta}+\frac{1}{A\left(M_{N}\right)-B\left(M_{N}\right) \cos 2 \theta}\right]\right) f^{e \mu}\left(M_{N}\right),
\end{aligned}
$$

where $\beta=\frac{1}{128 \pi^{3}} \frac{M_{W_{L}}^{8}}{M_{W_{R}}^{4}} G_{F}^{2}\left(V_{M_{1}}^{\mathrm{CKM}} V_{M_{2}}^{\mathrm{CKM}}\right)^{2} f_{M_{1}}^{2} f_{M_{2}}^{2}$, and we consider $M_{N_{1}}=M_{N_{2}}=M_{N}$. The functions $f^{e e}$, $f^{\mu \mu}$, and $f^{e \mu}$ can be identified from Eq. (A11). The first two terms are due to the individual $N_{1,2}$ contributions in the decay amplitude. The term in the partial decay width expressions proportional to $\cos 2 \phi \sin ^{2} 2 \theta$ is due to the interference of the $N_{1,2}$ contributions.

These complicated expressions simplify for RH neutrinos with a mass $M_{N} \sim$ few $\mathrm{GeV}$ range, where the mass of the final-state leptons can be ignored in evaluating the total decay width of RH neutrinos. Using Eq. (35), and Eqs. (36), (37), and (38), we find that for RH neutrinos with masses $\sim$ few $\mathrm{GeV}$, the LNV partial width of the parent meson $M_{1}$ (such as a $B$ meson) has the following simplified dependencies on the mixing angle and phase:

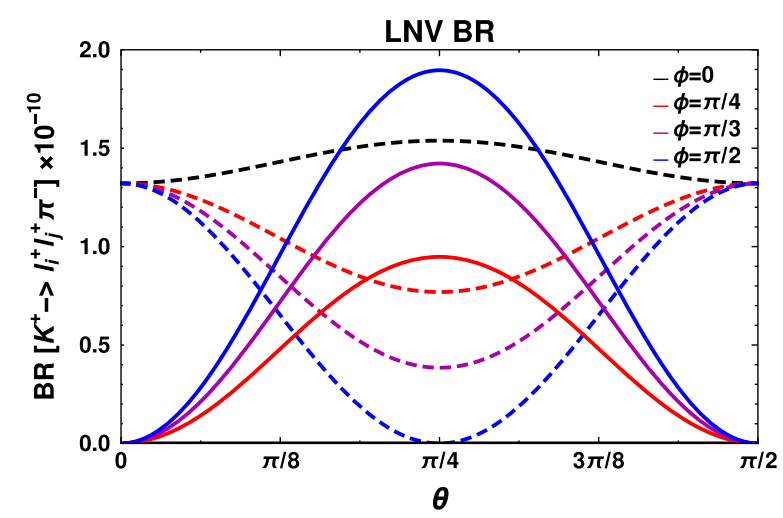

$$
\Gamma_{i j}^{\mathrm{LNV}} \propto \begin{cases}\left(1-\sin ^{2} 2 \theta \sin ^{2} \phi\right), & \text { for } i=j \\ \left(\sin ^{2} 2 \theta \sin ^{2} \phi\right), & \text { for } i \neq j\end{cases}
$$

In the above equation $i, j$ can be either $e$ or $\mu$. Similar expressions for the $l l j j$ production cross section at the LHC were obtained in Ref. [41].

The branching ratios of the different LNV modes of the parent meson $M_{1}$ are then given by

$$
\operatorname{Br}\left(M_{1}^{+} \rightarrow l_{i}^{+} l_{j}^{+} M_{2}^{-}\right)=\frac{\Gamma_{i j}^{\mathrm{LNV}}}{\Gamma_{M_{1}^{+}}},
$$

where $\Gamma_{M_{1}^{+}}$is the total decay width of the parent meson. In Fig. 5 we show the branching ratios of the LNV meson

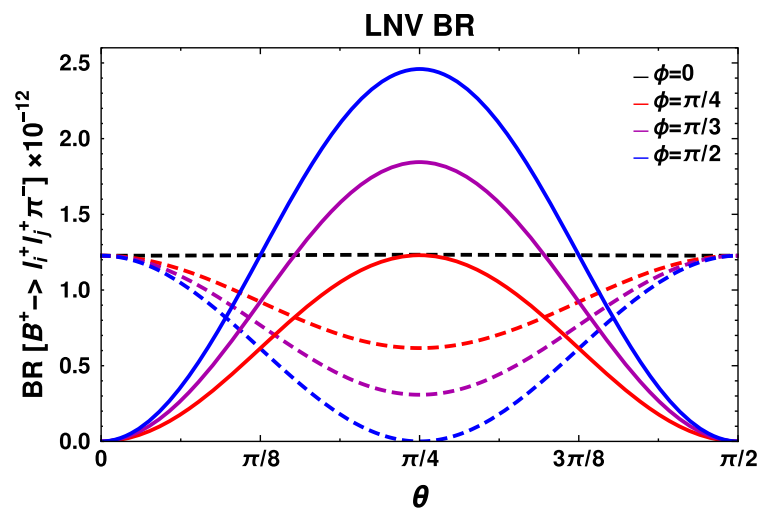

FIG. 5. Branching ratio of LNV meson decay to same-flavor $\left(e^{+}, e^{+}\right)$and different-flavor $\left(e^{+}, \mu^{+}\right)$final states along with a pion $\left(\pi^{+}\right)$. The plot in the left panel is for the kaon $\left(K^{+}\right)$decay, and the plot in the right panel is for $B$-meson $\left(B^{+}\right)$decay. The branching ratio is not constant; rather, it exhibits constructive and destructive interference effects for different values of $\theta$ and $\phi$. The dotted line and solid line correspond to the $e^{+} e^{+}$and $e^{+} \mu^{+}$mode, respectively. For $K^{+}$decay we consider $M_{N} \simeq 0.38 \mathrm{GeV}$ and $M_{W_{R}}=22 \mathrm{TeV}$, and for $B^{+}$meson decay we consider $M_{N} \simeq 2 \mathrm{GeV}$ and $M_{W_{R}}=5 \mathrm{TeV}$. 

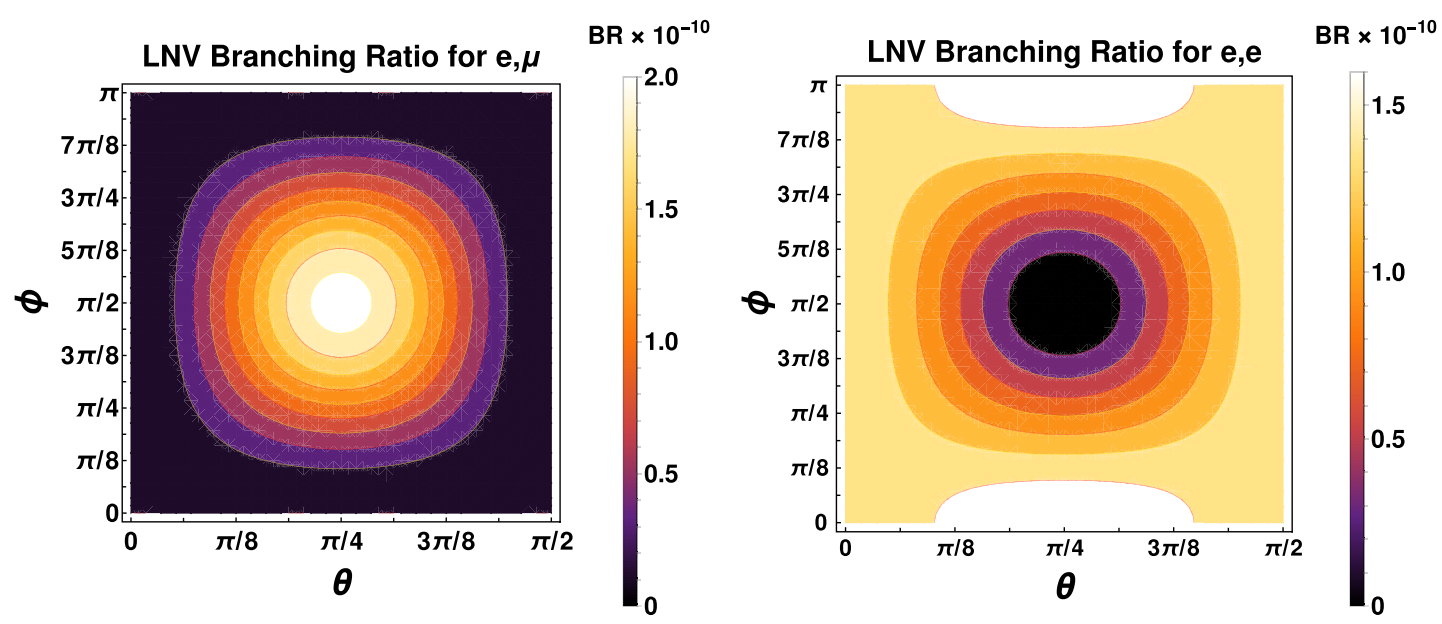

FIG. 6. Left: variation of the branching ratio of LNV kaon decay $K^{+} \rightarrow e^{+} \mu^{+} \pi^{-}$with the variation of the angle and phase $(\theta, \phi)$. The final-state leptons have the same charge and different flavors. Right: the same but for final states with same-charge and same-flavor leptons, $K^{+} \rightarrow e^{+} e^{+} \pi^{-}$. The masses of the RH neutrino and RH gauge bosons are $M_{N_{1}} \simeq M_{N_{2}} \simeq 0.38 \mathrm{GeV}$ and $M_{W_{R}}=22 \mathrm{TeV}$, respectively. The maximum values of the branching ratios are $\mathcal{O}\left(10^{-10}\right)$, consistent with the current experimental bounds.

decays $K^{+} / B^{+} \rightarrow e^{+} e^{+} \pi^{-}$and $K^{+} / B^{+} \rightarrow e^{+} \mu^{+} \pi^{-}$vs mixing angle $\theta$ for different values of $\phi$. The left panel corresponds to the kaon decay. We consider the mass of the RH gauge boson as $M_{W_{R}}=22 \mathrm{TeV}$, and the mass of the heavy neutrino as $M_{N} \simeq 0.38 \mathrm{GeV}$. In the right panel we show the LNV decay of the $B^{+}$meson. For this case, we consider the RH gauge boson mass to be $M_{W_{R}}=5 \mathrm{TeV}$, and the mass of the heavy neutrino is $M_{N} \simeq 2 \mathrm{GeV}$. This choice of RH neutrino and RH gauge boson mass, which is relevant for $B^{+}$study, is unconstrained from $K^{+}$-meson decays. The branching ratios derived for these benchmark mass points are consistent with the experimental limits $\operatorname{Br}\left(K^{+} \rightarrow e^{+} e^{+} \pi^{-}, e^{+} \mu^{+} \pi^{-}\right)<(2.2,5.0) \times 10^{-10} \quad$ and $\operatorname{Br}\left(B^{+} \rightarrow e^{+} e^{+} \pi^{-}, e^{+} \mu^{+} \pi^{-}\right)<(2.3,15.0) \times 10^{-8}$, respectively [56]. The figures confirm the angular dependencies of the branching ratios of the LNV decay process. The solid and dashed lines correspond to the $e^{+} \mu^{+}$and $e^{+} e^{+}$ channels. Note that the $e^{+} e^{+}$and $e^{+} \mu^{+}$channels have complimentary nature with respect to the angular variables. This can be further highlighted by a contour plot in the $\theta-\phi$ plane. Figure 6 shows the variation of the LNV branching ratios of the $K^{+} \rightarrow e^{+} e^{+} \pi^{-}$and $K^{+} \rightarrow e^{+} \mu^{+} \pi^{-}$ processes for different values of mixing angle $\theta$ and phase $\phi$. With two degenerate heavy neutrinos, the branching ratios of the above processes exhibit constructive and destructive interference, as is clearly evident from the figures. The white region in the right panel of Fig. 6 occurs as for $\theta=\pi / 4$, and $\phi \sim 0, \pi$, the LNV branching ratio to the $e^{+} e^{+}$channel shows a maxima (see left panel of Fig. 5). A different value of the RH gauge boson mass will simply give an overall scaling in the branching ratios and will not change the nature of Fig. 5. For the $B^{+}$meson, the plots are very similar. Hence, we do not show them explicitly.

\section{B. LNC}

The parent meson can decay via LNC processes $M_{1}^{+} \rightarrow l_{i}^{+} l_{j}^{-} M_{2}^{+}$. The RH neutrino states $N_{1,2}$ will mediate these processes. Considering the general form of the $N_{i}$ decay width as given in Eqs. (33) and (34), the LNC decay rate can be calculated. For the $e e$ and $\mu \mu$ channels the $N$ mediated decay rate is given by

$$
\begin{aligned}
\Gamma_{e e}^{\mathrm{LNC}}= & \pi \frac{\beta}{M_{N}}\left(\frac{\cos ^{4} \theta}{A\left(M_{N}\right)+B\left(M_{N}\right) \cos 2 \theta}+\frac{\sin ^{4} \theta}{A\left(M_{N}\right)-B\left(M_{N}\right) \cos 2 \theta}+\frac{1}{4} \sin ^{2} 2 \theta\right. \\
& \left.\times\left[\frac{1}{A\left(M_{N}\right)+B\left(M_{N}\right) \cos 2 \theta}+\frac{1}{A\left(M_{N}\right)-B\left(M_{N}\right) \cos 2 \theta}\right]\right) h^{e e}\left(M_{N}\right), \\
\Gamma_{\mu \mu}^{\mathrm{LNC}}= & \pi \frac{\beta}{M_{N}}\left(\frac{\sin ^{4} \theta}{A\left(M_{N}\right)+B\left(M_{N}\right) \cos 2 \theta}+\frac{\cos ^{4} \theta}{A\left(M_{N}\right)-B\left(M_{N}\right) \cos 2 \theta}+\frac{1}{4} \sin ^{2} 2 \theta\right. \\
& \left.\times\left[\frac{1}{A\left(M_{N}\right)+B\left(M_{N}\right) \cos 2 \theta}+\frac{1}{A\left(M_{N}\right)-B\left(M_{N}\right) \cos 2 \theta}\right]\right) h^{\mu \mu}\left(M_{N}\right),
\end{aligned}
$$



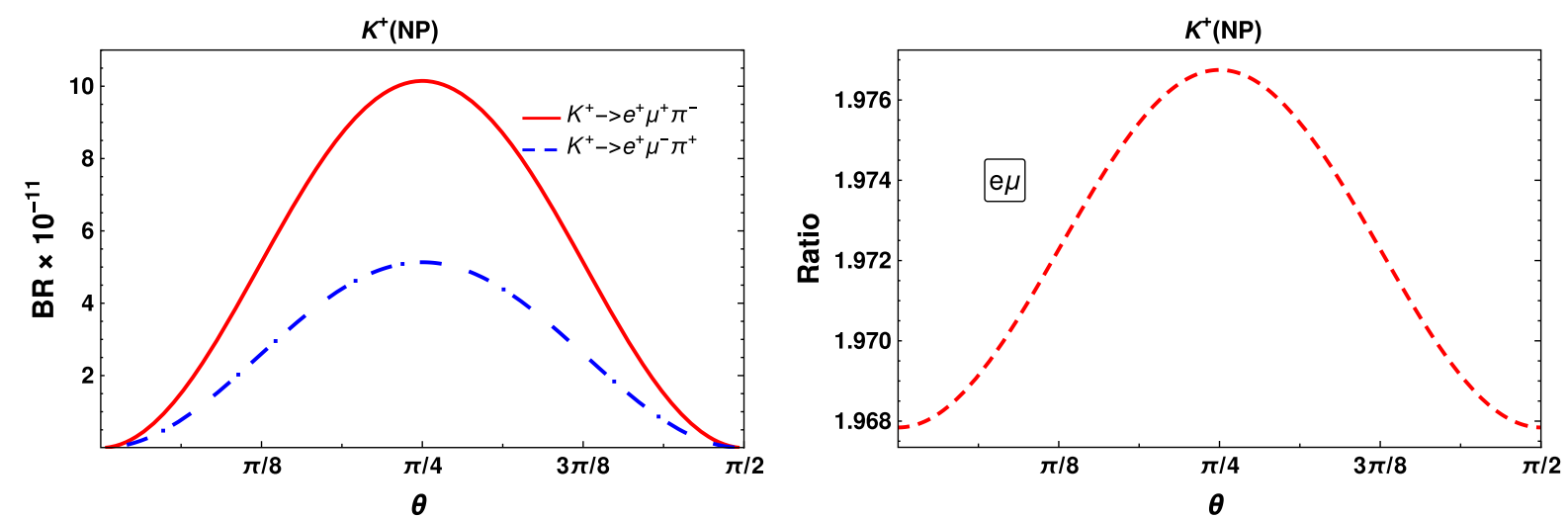

FIG. 7. Left: branching ratios of $K^{+} \rightarrow e^{+} \mu^{+} \pi^{-}$and $e^{+} \mu^{-} \pi^{+}$vs angle $\theta$ for nondegenerate RH neutrino masses considering $\frac{\delta M}{\Gamma_{N}} \sim 10$. Right: variation of the ratio of the two branching ratios vs mixing angle $\theta$. The results for $B^{+}$decays are similar, and hence we do not show them explicitly.

and for the $e \mu$ channel, the decay rate has the following form:

$$
\begin{aligned}
\Gamma_{e \mu}^{\mathrm{LNC}}= & \pi \frac{\beta}{M_{N}}\left(\frac{\sin ^{2} 2 \theta}{4\left(A\left(M_{N}\right)+B\left(M_{N}\right) \cos 2 \theta\right)}+\frac{\sin ^{2} 2 \theta}{4\left(A\left(M_{N}\right)-B\left(M_{N}\right) \cos 2 \theta\right)}-\frac{1}{4} \sin ^{2} 2 \theta\right. \\
& \left.\times\left[\frac{1}{A\left(M_{N}\right)+B\left(M_{N}\right) \cos 2 \theta}+\frac{1}{A\left(M_{N}\right)-B\left(M_{N}\right) \cos 2 \theta}\right]\right) h^{e \mu}\left(M_{N}\right) .
\end{aligned}
$$

The functions $h^{e e}, h^{\mu \mu}$, and $h^{e \mu}$ can be identified from Eq. (A15). Note that the functions $h^{i j}$ in the above expressions are related to $f^{i j}$ of the previous section as $M_{N}^{2} f^{i j}=h^{i j}$. We use the same values for the RH neutrino and RH gauge boson masses that we considered for LNV processes. Using Eq. (43), and after simplification, the LNC partial decay width for different lepton flavor $e \mu$ becomes zero for degenerate RH neutrino masses $M_{N_{1}}=M_{N_{2}}$. This however becomes nonzero if we consider nondegenerate masses of RH neutrinos. In the left panel of Fig. 7, we show the branching ratios of $K^{+} \rightarrow$ $e^{+} \mu^{+} \pi^{-}, e^{+} \mu^{-} \pi^{+}$for $\frac{\delta M}{\Gamma_{N}} \sim 10$. In the right panel of Fig. 7, we show the ratio of the two branching ratios for the $e \mu$ channel. As can be seen, due to the interference between the two RH neutrino states the ratio of the LNV and LNC branching ratios differs from unity. Using Eqs. (41) and (42), the partial width for same-flavor leptons turns out to be large, and is almost independent of the mixing angle $\theta$. We find that the contributions of RH-neutrino-mediated diagrams in the branching ratio of the same-flavor LNC decay mode of $K^{+}\left(K^{+} \rightarrow e^{+} e^{-} \pi^{+}\right)$and $B^{+}$ $\left(B^{+} \rightarrow e^{+} e^{-} \pi^{+}\right)$mesons are $\mathcal{O}\left(10^{-10}\right)$ and $\mathcal{O}\left(10^{-12}\right)$, respectively. As these same-flavor LNC decay modes also contain large SM contributions $\left(\mathrm{BR} \sim 10^{-7} / 10^{-8}\right)$ along with the order-of-magnitude suppressed new physics contribution, we do not show them here. In Sec. V we elaborate on the large LNC branching ratio for the $e \mu$ case, while using a $3 \times 3 K_{R}$ matrix.

\section{Effect of interference for $\mathrm{RH}$ neutrino and $\mathrm{RH}$ gauge boson masses}

In Fig. 8, we represent the effect of interference in the $M_{N}-M_{W_{R}}$ mass plane, considering LNV modes. We consider two different decay modes: $K^{+} / B^{+} \rightarrow e^{+} \mu^{+} \pi^{-}$ and $K^{+} / B^{+} \rightarrow e^{+} e^{+} \pi^{-}$. The solid red line in the left panel has been derived by assuming only one generation RH neutrino state $N_{1}$. This corresponds to the present experimental limit of the branching ratio $K^{+} \rightarrow e^{+} \mu^{+} \pi^{-}$, which is $\operatorname{Br}\left(K^{+} \rightarrow e^{+} \mu^{+} \pi^{-}\right)<5 \times 10^{-10}$ [56]. The blue dashed and blue solid lines correspond to the two-generation RH neutrino scenario where both $N_{1,2}$ can be produced on shell from parent meson decay. The subsequent decays of $N_{1,2}$ lead to the same final state $e^{+} \mu^{+} \pi^{-}$with the same value of the branching ratio. We have set the mixing angle and phase as $\theta=\pi / 4, \phi=\pi / 2+0.1$ for the blue dashed line. The solid blue line corresponds to the two-generation scenario, with $\theta=\pi / 4, \phi=0.1$, and again corresponds to the experimental limit on the branching ratio. ${ }^{1}$ The black dashed line and black solid line correspond to a value of $10^{-12}$ for the branching ratios of $K^{+} \rightarrow e^{+} \mu^{+} \pi^{-}$and $K^{+} \rightarrow e^{+} e^{+} \pi^{-}$. In the right panel, we show the result for $B$ meson decays $B^{+} \rightarrow e^{+} e^{+} \pi^{-} / e^{+} \mu^{+} \pi^{-}$. The two kinks occur as new decay modes of the $\mathrm{RH}$ neutrinos into

\footnotetext{
${ }^{1}$ We have not considered $\phi$ to be exactly $\pi / 2$ or 0 as the theoretical branching ratio will be exactly zero, and hence it is not possible to derive any kind of bound in the $M_{N}-M_{W_{R}}$ plane.
} 

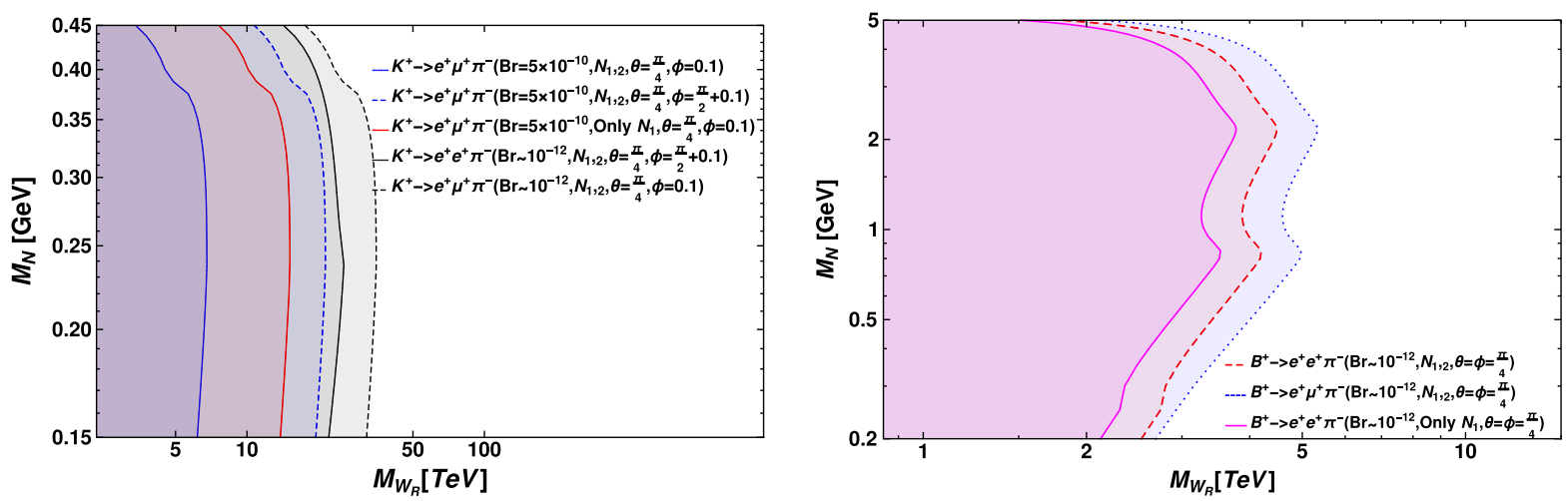

FIG. 8. Sensitivity of the RH neutrino mass $M_{N}$ and the RH gauge boson mass $M_{W_{R}}$ from the LNV processes $K^{+} \rightarrow e^{+} e^{+} \pi^{-}$, $K^{+} \rightarrow e^{+} \mu^{+} \pi^{-}$(left panel) and $B^{+} \rightarrow e^{+} \mu^{+} \pi^{-}, e^{+} e^{+} \pi^{-}$(right panel). For the $K^{+}$decay mode in the upper panel, the red solid line corresponds to the one-generation scenario, while the blue dashed line represents the two-generation scenario with constructive interference, $\theta=\pi / 4, \phi \sim 0, \pi / 2$. The blue solid line represents the two-generation scenario with destructive interference. The black solid and dashed lines represent $\mathrm{Br} \sim 10^{-12}$. The figure in the right panel indicates the future sensitivity of $\mathrm{LNV} B^{+}$meson decay with $\mathrm{Br} \sim 10^{-12}$.

a lepton associated with a $\rho$ and $D$ meson open up in these mass ranges. The solid pink line in the right panel has been again derived by assuming only the one-generation $\mathrm{RH}$ neutrino state $N_{1}$, where as the blue dotted and red dashed lines stand for the two-generation RH neutrino scenario. For the $B$-meson case, we consider the future sensitivity on the branching ratio to be $\mathcal{O}\left(10^{-12}\right){ }^{2}$ Note that the limit on $W_{R}$ can be significantly lowered in the presence of destructive interference as it leads to a natural suppression of the LNV branching ratio. Nonobservation of any LNV signal for the LRSM could signify interference effects due to more than one generation of RH neutrinos.

\section{Asymmetries in LNV and LNC processes}

The nontrivial phase $\phi$, along with the interference effect between $N_{1,2}$ contributions result in asymmetries in LNV and LNC processes. Before discussing the different asymmetries and the effect of interference, we first quantify the effect of a small mass splitting on the LNV and LNC meson decays. The obtained expressions will be used in deriving the expressions of $C P$ asymmetries. We represent the average mass of the two RH neutrinos as $M_{N}$ and the mass splitting as $\delta M$. Therefore, we follow the convention $M_{N_{1}}=M_{N}-\frac{\delta M}{2}$ and $M_{N_{2}}=M_{N}+\frac{\delta M}{2}$. We assume the following conditions are realized:

$$
\delta M \ll M_{N} \text { and } \delta M<\Gamma_{N_{1}}, \Gamma_{N_{2}} .
$$

With the above set of approximations, the LNV and LNC meson decay rates for a nontrivial mixing matrix $K_{R}$ can be written as follows:

$$
\begin{aligned}
\Gamma_{i j}^{\mathrm{LNV},++}= & \left(1-\frac{\delta_{i j}}{2}\right) \pi \beta\left(\left|\left(K_{R}\right)_{1 i}\right|^{2}\left|\left(K_{R}\right)_{1 j}\right|^{2} \frac{\left(M_{N}-\frac{\delta M}{2}\right)}{\Gamma_{N_{1}}} f_{i j}\left(M_{N}-\frac{\delta M}{2}\right)\right. \\
& +\left|\left(K_{R}\right)_{2 i}\right|^{2}\left|\left(K_{R}\right)_{2 j}\right|^{2} \frac{\left(M_{N}+\frac{\delta M}{2}\right)}{\Gamma_{N_{2}}} f_{i j}\left(M_{N}+\frac{\delta M}{2}\right)+\frac{\left(M_{N}-\frac{\delta M}{2}\right)\left(M_{N}+\frac{\delta M}{2}\right)}{M_{N}} \\
& \left.\times \operatorname{Re}\left[\left(K_{R}\right)_{1 i}\left(K_{R}\right)_{1 j}\left(K_{R}\right)_{2 i}^{*}\left(K_{R}\right)_{2 j}^{*}\left(\frac{1-4 \mathrm{i} \frac{\delta M}{\Gamma_{N_{1}}}}{\Gamma_{N_{1}}\left(1+4 \frac{\delta M^{2}}{\Gamma_{N_{1}}^{2}}\right)}+\frac{1-4 \mathrm{i} \frac{\delta M}{\Gamma_{N_{2}}}}{\Gamma_{N_{2}}\left(1+4 \frac{\delta M^{2}}{\Gamma_{N_{2}}^{2}}\right)}\right)\right] f_{i j}\left(M_{N}\right)+(i \Leftrightarrow j)\right) .
\end{aligned}
$$

The charge-conjugate process $M_{1}^{-} \rightarrow \ell_{i}^{-} \ell_{j}^{-} \pi^{+}$can be obtained by replacing $K_{R}$ with $K_{R}^{*}$ in Eq. (45):

$$
\Gamma_{i j}^{\mathrm{LNV},--}=\Gamma_{i j}^{\mathrm{LNV},++}\left(K_{R} \rightarrow K_{R}^{*}\right)
$$

\footnotetext{
${ }^{2}$ The current bounds for the $B$-meson LNV semileptonic decays are $\mathrm{BR}\left(B^{+} \rightarrow e^{+} e^{+} \pi^{-}\right) \leq 2.3 \times 10^{-8}$ and $\mathrm{BR}\left(B^{+} \rightarrow e^{+} \mu^{+} \pi^{-}\right) \leq$ $1.5 \times 10^{-7}[56]$.
} 
For LNC, this takes the following form:

$$
\begin{aligned}
\Gamma_{i j}^{\mathrm{LNC}}= & \pi \beta\left(\left|\left(K_{R}\right)_{1 i}\right|^{2}\left|\left(K_{R}\right)_{1 j}\right|^{2} \frac{1}{\Gamma_{N_{1}}\left(M_{N}-\frac{\delta M}{2}\right)} h_{i j}\left(M_{N}-\frac{\delta M}{2}\right)+\left|\left(K_{R}\right)_{2 i}\right|^{2}\left|\left(K_{R}\right)_{2 j}\right|^{2} \frac{1}{\Gamma_{N_{2}}\left(M_{N}+\frac{\delta M}{2}\right)} h_{i j}\left(M_{N}+\frac{\delta M}{2}\right)\right. \\
& \left.+\frac{1}{M_{N}} \operatorname{Re}\left[\left(K_{R}\right)_{1 i}^{*}\left(K_{R}\right)_{1 j}\left(K_{R}\right)_{2 i}\left(K_{R}\right)_{2 j}^{*}\left(\frac{1-4 \mathrm{i} \frac{\delta M}{\Gamma_{N_{1}}}}{\Gamma_{N_{1}}\left(1+4 \frac{\delta M^{2}}{\Gamma_{N_{1}}^{2}}\right)}+\frac{1-4 \mathrm{i} \frac{\delta M}{\Gamma_{N_{2}}}}{\Gamma_{N_{2}}\left(1+4 \frac{\delta M^{2}}{\Gamma_{N_{2}}^{2}}\right)}\right)\right] h_{i j}\left(M_{N}\right)\right) .
\end{aligned}
$$

The interference between the two RH neutrino $N_{1}$ and $N_{2}$ contributions along with a nonzero $C P$ phase $\phi$ can lead to a difference between the LNV process $M_{1}^{+} \rightarrow \ell_{i}^{+} \ell_{j}^{+} \pi^{-}$and the charge-conjugate process $M_{1}^{-} \rightarrow \ell_{i}^{-} \ell_{j}^{-} \pi^{+}$. This can be quantified by the following definition:

$$
A_{C P}^{i j}=\frac{\Gamma_{i j}^{\mathrm{LNV},++}-\Gamma_{i j}^{\mathrm{LNV},--}}{\Gamma_{i j}^{\mathrm{LNV},++}+\Gamma_{i j}^{\mathrm{LNV},--}} \quad(i, j=e, \mu) .
$$

Using Eqs. (45), (46), and (32) and the nearly degenerate limit of the masses and decay widths, $M_{N_{1}} \approx M_{N_{2}} \equiv M_{N}{ }^{3}$ $\Gamma_{N_{1}} \approx \Gamma_{N_{2}} \equiv \Gamma_{N}, A_{C P}^{i j}$ takes the following simple forms:

$$
A_{C P}^{i i} \approx \frac{4 x \sin 2 \phi \sin ^{2} 2 \theta}{\left(1+4 x^{2}\right)\left(2 \cos ^{4} \theta+2 \sin ^{4} \theta\right)+\cos 2 \phi \sin ^{2} 2 \phi},
$$

with $i=e, \mu$,

$$
A_{C P}^{i j} \approx \frac{-4 x \sin 2 \phi}{\left(1+4 x^{2}-\cos 2 \phi\right)}, \quad \text { where } i \neq j
$$

In the above, $x=\frac{\delta M}{\Gamma_{N}}$. For a fixed $\theta$ and $x$, the above equations lead to a maximum value of $A_{C P}$ if $\phi=\frac{\pi}{4}$. In Fig. 9, we show the variation of the LNV-CP asymmetry as a function of $\frac{\delta M}{\Gamma_{N}}$ for the channels $e^{+} e^{+}, \mu^{+} \mu^{+}$, and $e^{+} \mu^{+}$, where we fix $\theta=\frac{\pi}{4}$ and $\phi=\frac{\pi}{4}$. We see from this figure that $A_{C P}^{e e \text { or } \mu \mu}$ and $A_{C P}^{e \mu}$ have complementary behavior as a function of $\frac{\delta M}{\Gamma_{N}}$. In order to discuss the impact of the $C P$ phase and mixing angle on the LNV width, we further define one more asymmetry $\tilde{R}$ as

$\tilde{R}_{i j}=\frac{\Gamma_{K^{+} \rightarrow \ell_{i}^{+} \ell_{i}^{+} \pi^{-}}^{\mathrm{LNV}}-\Gamma_{K^{+} \rightarrow \ell_{i}^{+} \ell_{j}^{+} \pi^{-}}^{\mathrm{LNV}}}{\Gamma_{K^{+} \rightarrow \ell_{i}^{+} \ell_{i}^{+} \pi^{-}}^{\mathrm{LNV}}+\Gamma_{K^{+} \rightarrow \ell_{i}^{+} \ell_{j}^{+} \pi^{-}}^{\mathrm{LNV}}}, \quad$ where $i \neq j$.

Figure 10 shows the variation of $\tilde{R}_{e \mu}$ for different values of mixing angle $\theta$ and phase $\phi$. It is evident from this figure that $\tilde{R}_{e \mu}$ varies between $[-1: 1]$. Note that $\left|\tilde{R}_{e \mu}\right|$ have complimentary nature with respect to the angular variables and their analytical expressions can be found in

\footnotetext{
${ }^{3}$ The mass difference $\delta M \neq 0$; however, $\delta M \ll M_{N}$.
}

Appendix B. Similar results can also be obtained for $B$ decays. This implies that the two asymmetries $A_{C P}$ and $\tilde{R}_{e \mu}$ do not contain any SM contribution, and hence serve as a clear indication of new physics.

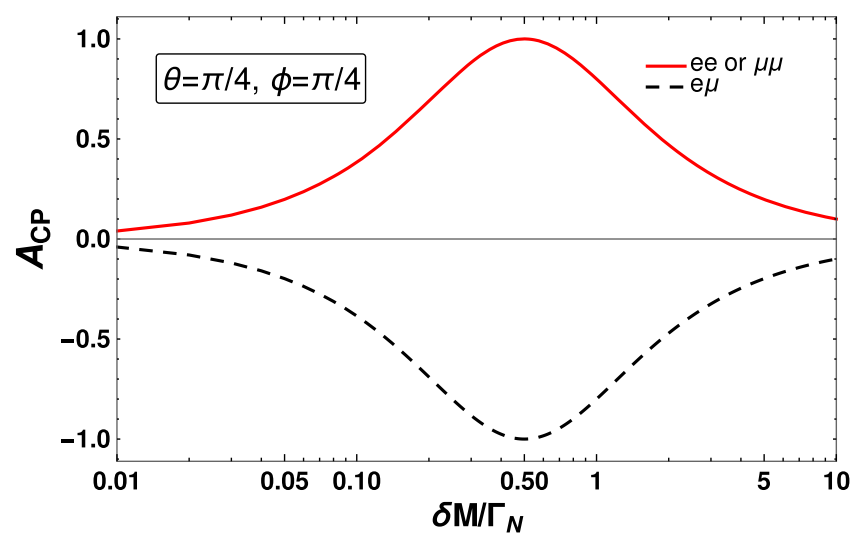

FIG. 9. Variation of $A_{C P}$ as a function of $\frac{\delta M}{\Gamma_{N}}$, for $\theta=\frac{\pi}{4}$ and $\phi=\frac{\pi}{4}$. The red solid line represents the $e^{+} e^{+}$or $\mu^{+} \mu^{+}$channel, whereas the black dashed line represents the $e^{+} \mu^{+}$channel. For this figure, we consider $\mathrm{K}^{+}$as the parent meson. For the $B^{+}$meson the figure is similar, and hence we do not show this explicitly.

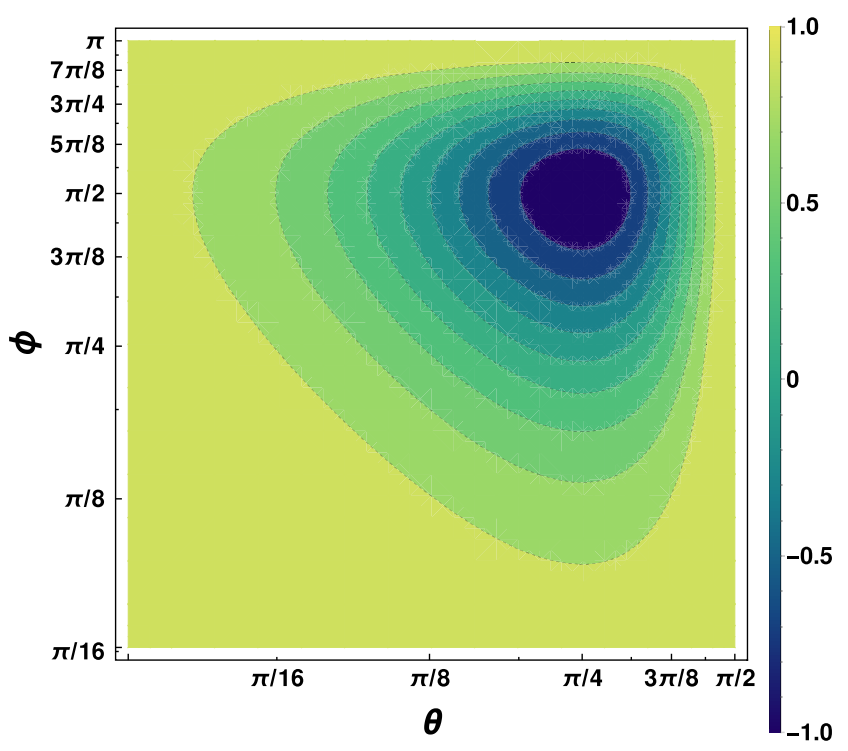

FIG. 10. Variation of $\tilde{R}_{e \mu}$ with the variation of angle and phase $(\theta, \phi) . \tilde{R}$ varies between $[-1: 1]$. 

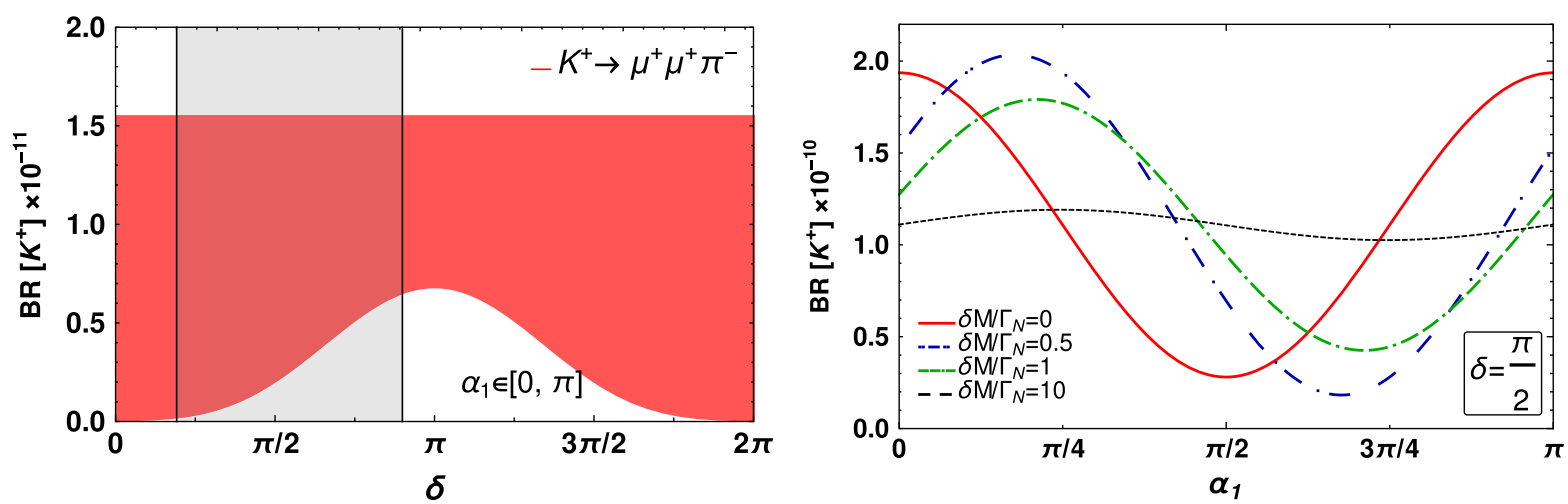

FIG. 11. Left: variation of the branching ratio of the $K^{+} \rightarrow \mu^{+} \mu^{+} \pi^{-}$decay mode with the variation of the $C P$-violating phase $\delta$. The Majorana phases $\alpha_{1}$ are varied in the denoted range. The shaded region is disallowed from the recent results from T2K. Note that the excluded region from T2K for $\delta$ is given with the convention $[-\pi: \pi]$, which we have translated into the range $[0: 2 \pi]$ to be consistent with our convention. See text for more details. Right: variation of the branching ratio of the $K^{+} \rightarrow e^{+} e^{+} \pi^{-}$decay mode with the variation of the Majorana phase $\alpha_{1}$. The red solid, blue dash-double-dotted, green dash-dotted, and black dashed lines represent four different $\frac{\delta M}{\Gamma_{N}}$ ratios $(0,0.5,1,10)$.

\section{RESULT FOR TWO GENERATION WITH THE FULL $3 \times 3$ MIXING MATRIX $K_{R}$}

In the previous section, we considered a simple form of the $K_{R}$ matrix, which contains only one angle and one phase. In general, the mixing matrix $K_{R}$ is a $3 \times 3$ unitary matrix, with more number of parameters. We consider a special scenario where the $K_{R}$ matrix is identical to the
PMNS mixing matrix in the light neutrino sector. The additional $C P$ phases can give sizable contributions to both the LNV and LNC meson decays; in particular, they can give a different result in the LNC $e \mu$ mode as compared to the previous scenario where we considered a simple form for $K_{R}$. We consider the following parametrization of $K_{R}$ :

$$
K_{R}=\left[\begin{array}{ccc}
c_{12} c_{13} & -c_{23} s_{12}-e^{-i \delta} c_{12} s_{13} s_{23} & -e^{-i \delta} c_{12} c_{23} s_{13}+s_{12} s_{23} \\
e^{-i \alpha_{1}} c_{13} s_{12} & e^{-i \alpha_{1}} c_{12} c_{23}-e^{-i\left(\alpha_{1}+\delta\right)} s_{12} s_{13} s_{23} & -e^{-i\left(\alpha_{1}+\delta\right)} c_{23} s_{12} s_{13}-e^{-i \alpha_{1}} c_{12} s_{23} \\
e^{-i \alpha_{2}} s_{13} & e^{-i\left(\alpha_{2}+\delta\right)} c_{13} s_{23} & e^{-i\left(\alpha_{2}+\delta\right)} c_{13} c_{23}
\end{array}\right]
$$

where $s_{12}, s_{13}, s_{23}$ refer to $\sin \theta_{12}, \sin \theta_{13}, \sin \theta_{23}$, respectively, and $\left(\alpha_{1}, \alpha_{2}\right) \in(0, \pi), \delta \in(0,2 \pi)$. Considering such a $3 \times 3$ matrix, the number of parameters increases and the result is much more complicated as compared to the former scenario.

Note that from Eq. (52), one would obtain Eq. (32) by considering $\theta_{13}, \theta_{23}=0, \alpha_{1}=\delta$, and $\alpha_{2}=0$. In Fig. 11 we show the variation of the LNV branching ratio $K^{+} \rightarrow$ $\mu^{+} \mu^{+} \pi^{-}$with the variation of the Dirac $C P$ phase $\delta$. We have checked that the RH neutrino contribution in the LNC branching ratio $K^{+} \rightarrow \mu^{+} \mu^{-} \pi^{+}$is independent of the Dirac phase $\delta$, and hence we do not show it explicitly. We consider best-fit values of the mixing angles $\theta_{12}, \theta_{13}, \theta_{23}$ for an inverted mass hierarchy [57], and vary the Majorana phases $\alpha_{1}$ in between 0 and $\pi$. As an illustrative example, we consider the mass splitting $\delta M=0$ and $M_{N}=0.38 \mathrm{GeV}$. We also show the regions of $\delta$ disallowed by the T2K neutrino oscillation experiment for an inverted mass hierarchy. The red region represents the LNV branching ratio, which clearly shows a nontrivial variation with respect to $\alpha_{1}$ and $\delta$. This is true that LNC mode should be independent of any Majorana phase. ${ }^{4}$ We further find that the decay mode $K^{+} \rightarrow e^{+} e^{+} \pi^{-}$depends only on the Majorana phase $\alpha_{1}$, and not on the Dirac phase $\delta$. The LNC mode with the same lepton flavor is also independent of the Dirac $C P$ phase, which happens due to the chosen form of $K_{R}$ in Eq. (52). This can be verified by substituting the explicit form of the $K_{R}$ matrix [Eq. (52)] into Eq. (45). In the right panel of Fig. 11 we show the variation of the LNV branching ratio $K^{+} \rightarrow e^{+} e^{+} \pi^{-}$with respect to the Majorana phase $\alpha_{1}$ for different $\frac{\delta M}{\Gamma_{N}}$ ratios. The RH neutrino contribution in the LNC

\footnotetext{
${ }^{4}$ Note that although the LNC decay modes do not depend on the Majorana phases, they can in principle depend on the Dirac phase $\delta$. We have verified that with our chosen $K_{R}$ matrix there is no dependency of $K^{+} \rightarrow \mu^{+} \mu^{-} \pi^{+}$on the Dirac CP phase, for $\delta M=0$.
} 

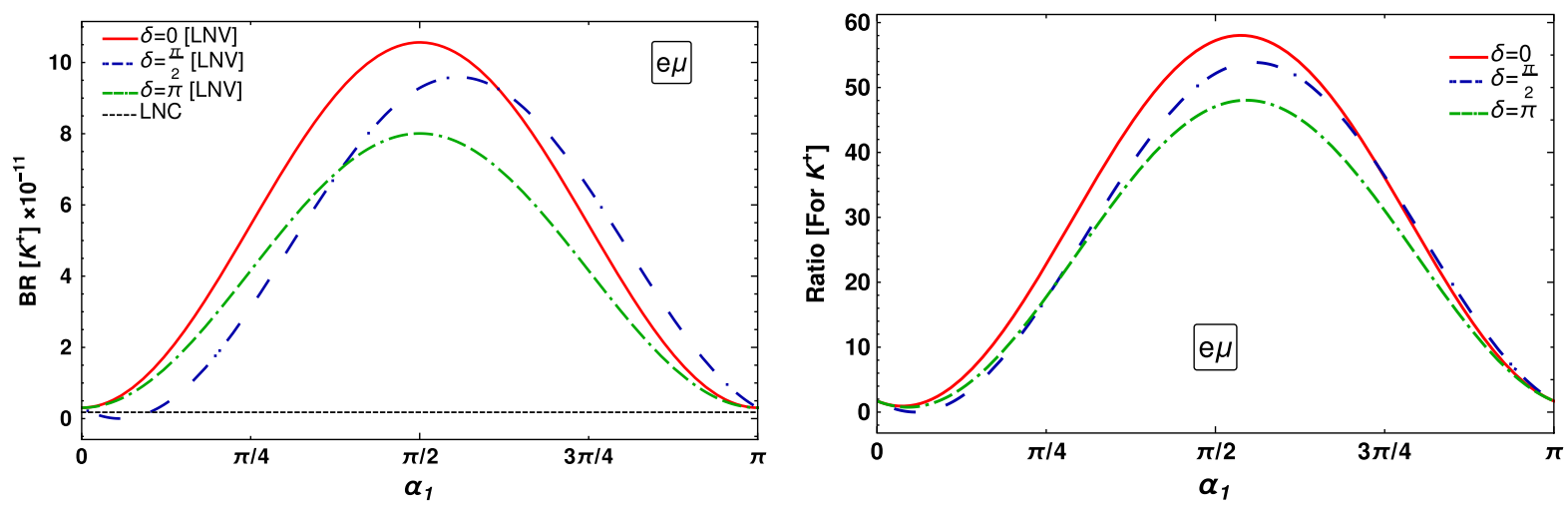

FIG. 12. Left: variation of the branching ratio of the $K^{+} \rightarrow e^{+} \mu^{+} \pi^{-}$and $K^{+} \rightarrow e^{+} \mu^{-} \pi^{+}$decay modes with the variation of the Majorana phase $\alpha_{1}$ and for different Dirac phases $\delta=\frac{\pi}{4}$ (solid red), $\frac{\pi}{2}$ (blue dash-double-dotted), and $\frac{3 \pi}{4}$ (green dash-dotted). Right: the ratio of the $\mathrm{LNV}$ and $\mathrm{LNC}$ branching ratios in the $e \mu$ mode.

branching ratio $K^{+} \rightarrow e^{+} e^{-} \pi^{+}$is independent of the Majorana phase, and hence we do not show it explicitly.

Finally, we elaborate on the LNC $е \mu$ channel, which we found to be vanishing for a simple form of $K_{R}$ [shown in Eq. (32)] and for degenerate RH neutrino masses. With the approximation $\delta M=0$ and $\Gamma_{N_{1}}=\Gamma_{N_{2}} \equiv \Gamma_{N}$, Eq. (47) can be simplified and the LNC decay rate $\Gamma_{e \mu}^{\mathrm{LNC}}$ is proportional to the following expression:

$$
\begin{aligned}
\tilde{\Gamma}_{e \mu}= & \left|\left(K_{R}\right)_{11}\right|^{2}\left|\left(K_{R}\right)_{12}\right|^{2}+\left|\left(K_{R}\right)_{21}\right|^{2}\left|\left(K_{R}\right)_{22}\right|^{2} \\
& +2 \operatorname{Re}\left[\left(K_{R}\right)_{11}^{*}\left(K_{R}\right)_{12}\left(K_{R}\right)_{21}\left(K_{R}\right)_{22}^{*}\right] .
\end{aligned}
$$

This means that for the preceding scenario, our choice of a $2 \otimes 2$ subblock of the $K_{R}$ matrix (32) as a unitary matrix leads to $\tilde{\Gamma}_{e \mu}=0$. This occurs due to the unitary properties of $K_{R}$. Hence, for this simplified scenario the ratio $\Gamma_{e \mu}^{\mathrm{LNV}} / \Gamma_{e \mu}^{\mathrm{LNC}}$ is ill defined. However, this changes if one considers the full $3 \otimes 3$ matrix, as in this case the $2 \otimes 2$ subblock itself is not unitary. We find that for the $3 \times 3$ case and using Eq. (52), for degenerate RH neutrinos, $\tilde{\Gamma}_{e \mu}=c_{13}^{2} s_{13}^{2} s_{23}^{2}$. This certainly is nonzero for the best-fit values of the PMNS mixing angles. We stress that in this case it is possible to define the ratio $\Gamma_{e \mu}^{\mathrm{LNV}} / \Gamma_{e \mu}^{\mathrm{LNC}}$ and the ratio can be large as the best-fit value of $s_{13}$ is small. In the right panel of Fig. 12 we show the variation of the LNV and LNC branching ratios $K^{+} \rightarrow e^{+} \mu^{+} \pi^{-}$and $K^{+} \rightarrow e^{+} \mu^{-} \pi^{+}$ with respect to the Majorana phase $\alpha_{1}$ for different choices of the Dirac $C P$ phase $\delta$. In the right panel we also show the ratio of the LNV and LNC modes. We see that the LNV mode $K^{+} \rightarrow e^{+} \mu^{+} \pi^{-}$depends on both the Majorana and Dirac phases even for $\delta M=0$. For degenerate RH neutrinos, the LNC $e \mu$ mode is independent of the Dirac phase (left panel). Contrary to the results obtained in Sec. IV B, the LNC $e \mu$ mode in this scenario has a large branching ratio $\sim 10^{-11}$. The ratio of the $\mathrm{LNV}$ and $\mathrm{LNC}$ modes in this case is order of magnitude large ( 40-60) compared to the earlier scenario, due to the relative suppression of the LNC rate for a small $\theta_{13}$.

\section{CONCLUSION}

The LRSM is one of the most appealing models that accommodates the embedding of $\mathrm{RH}$ neutrinos in a natural way. The model contains RH neutrinos, RH gauge bosons, and other BSM states that can offer distinctive experimental signatures. In this work, we explored the LNV and LNC semileptonic meson decays, mediated by the RH neutrinos and RH gauge bosons, and quantified the effect of interference in these decays due to the presence of at least two degenerate/quasidegenerate $\mathrm{RH}$ neutrino states. The $\mathrm{RH}$ neutrinos with masses in the range of $\mathrm{MeV}$ - few $\mathrm{GeV}$ can provide a resonance enhancement to these rates. We considered a few specific decay modes of the $K^{+}$and $B^{+}$mesons, $K^{+} / B^{+} \rightarrow e^{+} e^{+} \pi^{-}$and $K^{+} / B^{+} \rightarrow e^{+} \mu^{+} \pi^{-}, e^{+} \mu^{-} \pi^{+}$, and analyzed the effect of interference in detail. We considered two RH neutrinos to be degenerate/nearly degenerate in mass with their masses in the ranges $M_{N_{1}} \sim M_{N_{2}} \sim 380 \mathrm{MeV}$ and $M_{N_{1}} \sim M_{N_{2}} \sim 2 \mathrm{GeV}$, relevant for the LNV and LNC $K^{+}$and $B^{+}$-meson decays. In the case of a single generation of RH neutrinos, the LNV and LNC decay contributions are the same, leading to the ratio of these two to be unity. We found that in the presence of interference between the two RH neutrino contributions in the amplitude, the contributions of the RH neutrinos in the LNV and LNC decay rates can differ widely, which leads to the ratio to be different than unity.

We first considered a simplistic scenario where two RH neutrinos contribute to meson decay, with a simple RH neutrino mixing matrix consisting of one angle $\theta$ and one phase $\phi$. We showed that the LNV branching ratio-in particular, the interference term-depends on both the angle and phase. The RH neutrino contribution to the LNC mode only depends on the angle, however, and is insensitive to the phase. A few comments are in order: 
(1) The channels $K^{+} \rightarrow e^{+} e^{+} \pi^{-}$and $K^{+} \rightarrow e^{+} \mu^{+} \pi^{-}$ offer a complimentary nature in their predicted branching ratios. This holds true for other meson decays as well.

(2) We found that, overall, the LNV branching ratios $K^{+} / B^{+} \rightarrow e^{+} e^{+} \pi^{-}$have a large variation with respect to the variation in $\theta$ and $\phi$. The decay rates are highly suppressed due to destructive interference at $\phi \sim \pi / 2$ and $\theta \sim \pi / 4$. For both the processes, the predicted branching ratios can reach maximum $\mathcal{O}\left(10^{-10}\right), \mathcal{O}\left(10^{-12}\right)$ range with a $\mathrm{RH}$ gauge boson mass $22 \mathrm{TeV}$ and $5 \mathrm{TeV}$, respectively.

(3) For the different lepton flavor in the final states, $K^{+} \rightarrow e^{+} \mu^{-} \pi^{+}$mode, the LNC branching ratio is $\mathrm{Br} \sim 10^{-11}$ for nondegenerate $\mathrm{RH}$ neutrinos with $\frac{\delta M}{\Gamma_{N}} \sim 10$. The branching ratio for the LNV decay mode $K^{+} \rightarrow e^{+} \mu^{+} \pi^{-}$can be as high as $\mathcal{O}\left(10^{-10}\right)$.

We also explored a scenario where the mixing matrix in the RH neutrino sector has the same form as the PMNS mixing matrix in the neutrino sector. We considered the best-fit values of the neutrino mixing parameters and varied the phases. We found that the Majorana and Dirac phases in this case have a large impact on the branching ratio for the LNV channel $K^{+} \rightarrow \mu^{+} \mu^{+} \pi^{-}$, as well as $K^{+} \rightarrow e^{+} \mu^{+} \pi^{-}$. The same LNV decay mode but with $e^{+} e^{+}$in the final state is independent of the Dirac phase, and depends only on the Majorana phase. For the LNC modes there is no dependency on the Majorana $C P$ phase. Choosing degenerate $\mathrm{RH}$ neutrino masses, we found that the $e^{+} \mu^{-}$mode is also independent of the Dirac $C P$ phase. We further found that in this case the branching ratio of $K^{+} \rightarrow e^{+} \mu^{-} \pi^{+}$can be sizable, even for RH neutrinos with degenerate masses. The ratio between LNV and LNC decays now takes values over a wider range. Any future measurement of the ratio different than unity will indicate possible interference effects. Furthermore, observations of the LNV signature $K^{+} \rightarrow e^{+} \mu^{+} \pi^{-}$together with the LNC signature $K^{+} \rightarrow$ $e^{+} \mu^{-} \pi^{-}$will indicate a nontrivial form of the $K_{R}$ matrix. Hence, the LNV mode as well as the LNC mode with different lepton flavors can serve as a smoking gun signal for underlying new physics.

\section{ACKNOWLEDGMENTS}

S. M. acknowledges the support from Spanish Grant No. FPA2017-85216-P (AEI/FEDER, UE), PROMETEO/ 2018/165 (Generalitat Valenciana) and the Spanish Red Consolider MultiDark FPA2017-90566-REDC. The work of R. M. G. is supported by the Department of Science and Technology, India under Grant No. SR/S2/JCB-64/2007. M. M. and R. M. G. thank the Indo-French Centre for the Promotion of Advanced Research for the support (Project No: 6304-2).

\section{APPENDIX A: DETAILS OF LNV AND LNC CALCULATIONS}

\section{LNV}

Amplitude from the $N_{a}$ contribution:

$$
\mathcal{M}_{i j}^{\mathrm{LNV}, a}=2 G_{F}^{2} V_{M_{1}}^{\mathrm{CKM}} V_{M_{2}}^{\mathrm{CKM}} f_{M_{1}} f_{M_{2}}\left(\frac{M_{W_{L}}}{M_{W_{R}}}\right)^{4}\left(K_{R}^{*}\right)_{a i}\left(K_{R}^{*}\right)_{a j} M_{N_{a}} \frac{\bar{u}\left(k_{1}\right) \not \mid k_{3} P_{R} v\left(k_{2}\right)}{q^{2}-M_{N a}^{2}+i \Gamma_{N a} M_{N a}} .
$$

Amplitude for the leptonic exchange diagram:

$$
\begin{gathered}
\mathcal{M}_{j i}^{\mathrm{LNV}, a}=2 G_{F}^{2} V_{M_{1}}^{\mathrm{CKM}} V_{M_{2}}^{\mathrm{CKM}} f_{M_{1}} f_{M_{2}}\left(\frac{M_{W_{L}}}{M_{W_{R}}}\right)^{4}\left(K_{R}^{*}\right)_{a j}\left(K_{R}^{*}\right)_{a i} M_{N_{a}} \frac{\bar{u}\left(k_{2}\right) \not \not k_{3} P_{R} v\left(k_{1}\right)}{q^{2}-M_{N a}^{2}+i \Gamma_{N a} M_{N a}}, \\
\left|\mathcal{M}_{i j}^{\mathrm{LNV}, a}\right|^{2}=\kappa M_{N_{a}}^{2}\left(K_{R}^{*}\right)_{a i}\left(K_{R}^{*}\right)_{a j}\left(K_{R}\right)_{a i}\left(K_{R}\right)_{a j} \frac{\operatorname{Tr}\left[\left(\not k_{2}-m_{2}\right) \not k_{3} \not \not P_{L}\left(\not k_{1}+m_{1}\right) \not \not k_{3} P_{R}\right]}{\left(q^{2}-M_{N_{a}}^{2}\right)^{2}+\Gamma_{N_{a}}^{2} M_{N_{a}}^{2}}, \\
\left|\mathcal{M}_{j i}^{\mathrm{LNV}, a}\right|^{2}=\kappa M_{N_{a}}^{2}\left(K_{R}^{*}\right)_{a j}\left(K_{R}^{*}\right)_{a i}\left(K_{R}\right)_{a j}\left(K_{R}\right)_{a i} \frac{\operatorname{Tr}\left[\left(\not k_{1}-m_{1}\right) \not k_{3} \not \not p P_{L}\left(\not k_{2}+m_{2}\right) \not \not \not k_{3} P_{R}\right]}{\left(q^{2}-M_{N_{a}}^{2}\right)^{2}+\Gamma_{N_{a}}^{2} M_{N_{a}}^{2}},
\end{gathered}
$$

where $\kappa=4 G_{F}^{4}\left(V_{M_{1}}^{\mathrm{CKM}}\right)^{2}\left(V_{M_{2}}^{\mathrm{CKM}}\right)^{2} f_{M_{1}}^{2} f_{M_{2}}^{2}\left(\frac{M_{W_{L}}}{M_{W_{R}}}\right)^{8}$. The interference terms are

$$
\begin{aligned}
\left(\mathcal{M}_{i j}^{\mathrm{LNV}, a}\right)^{\dagger} \mathcal{M}_{i j}^{\mathrm{LNV}, b}= & \kappa M_{N_{a}} M_{N_{b}}\left(K_{R}\right)_{a i}\left(K_{R}\right)_{a j}\left(K_{R}^{*}\right)_{b i}\left(K_{R}^{*}\right)_{b j} \\
& \times \frac{\operatorname{Tr}\left[\left(\not k_{2}-m_{2}\right) \not k_{3} \not \not P_{L}\left(\not K_{1}+m_{1}\right) \not \not k_{3} P_{R}\right]}{\left(q^{2}-M_{N_{a}}^{2}-i \Gamma_{N_{a}} M_{N_{a}}\right)\left(q^{2}-M_{N_{b}}^{2}+i \Gamma_{N_{b}} M_{N_{b}}\right)},
\end{aligned}
$$




$$
\begin{aligned}
\left(\mathcal{M}_{j i}^{\mathrm{LNV}, a}\right)^{\dagger} \mathcal{M}_{j i}^{\mathrm{LNV}, b}= & \kappa M_{N_{a}} M_{N_{b}}\left(K_{R}\right)_{a j}\left(K_{R}\right)_{a i}\left(K_{R}^{*}\right)_{b j}\left(K_{R}^{*}\right)_{b i} \\
& \times \frac{\operatorname{Tr}\left[\left(\not \not_{1}-m_{1}\right) \not k_{3} \not p P_{L}\left(\not k_{2}+m_{2}\right) \not \not k_{3} P_{R}\right]}{\left(q^{2}-M_{N_{a}}^{2}-i \Gamma_{N_{a}} M_{N_{a}}\right)\left(q^{2}-M_{N_{b}}^{2}+i \Gamma_{N_{b}} M_{N_{b}}\right)} .
\end{aligned}
$$

The traces are

$$
\begin{aligned}
\operatorname{Tr}_{21}= & \operatorname{Tr}\left[\left(\not k_{2}-m_{2}\right) \not k_{3} \not p P_{L}\left(\not k_{1}+m_{1}\right) \not \not k_{3} P_{R}\right] \\
= & 2 k_{1} \cdot k_{2} k_{3} \cdot k_{3} p \cdot p-4 k_{1} \cdot p k_{2} \cdot p k_{3} \cdot k_{3} \\
& -4 k 1 \cdot k_{3} k_{2} \cdot k_{3} p \cdot p+8 k_{1} \cdot p k_{2} \cdot k_{3} k_{3} \cdot p, \\
\operatorname{Tr}_{12}= & \operatorname{Tr}\left[\left(\not k_{1}-m_{1}\right) \not k_{3} \not p P_{L}\left(\not k_{2}+m_{2}\right) \not \not k_{3} P_{R}\right] \\
= & 2 k_{1} \cdot k_{2} k_{3} \cdot k_{3} p \cdot p-4 k_{1} \cdot p k_{2} \cdot p k_{3} \cdot k_{3} \\
& -4 k_{1} \cdot k_{3} k_{2} \cdot k_{3} p \cdot p+8 k_{1} \cdot k_{3} k_{2} \cdot p k_{3} \cdot p .
\end{aligned}
$$

As the RH neutrinos are produced on shell and $\Gamma_{N_{a}} \ll M_{N_{a}}$, in our decay width calculation we can use the narrow-width approximation,

$\frac{1}{\left(q^{2}-M_{N_{a}}^{2}\right)^{2}+\Gamma_{N_{a}}^{2} M_{N_{a}}^{2}} \rightarrow \frac{\pi}{\Gamma_{N_{a}} M_{N_{a}}} \delta\left(q^{2}-M_{N_{a}}^{2}\right)$.
Using the narrow-width approximation, the product of the propagators in the interference term can be written as

$$
\begin{gathered}
\frac{1}{\left[\left(q^{2}-M_{N_{1}}^{2}\right)^{2}-i \Gamma_{N_{1}} M_{N_{1}}\right]\left[\left(q^{2}-M_{N_{2}}^{2}\right)^{2}+i \Gamma_{N_{2}} M_{N_{2}}\right]} \\
=-\frac{i\left(M_{N_{2}}^{2}-M_{N_{1}}^{2}\right) \pi \delta\left(q^{2}-M_{N_{2}}^{2}\right)}{\left(M_{N_{2}}^{2}-M_{N_{1}}^{2}\right)^{2}+\Gamma_{N_{1}}^{2} M_{N_{1}}^{2}} \\
+\frac{i\left(M_{N_{1}}^{2}-M_{N_{2}}^{2}\right) \pi \delta\left(q^{2}-M_{N_{1}}^{2}\right)}{\left(M_{N_{1}}^{2}-M_{N_{2}}^{2}\right)^{2}+\Gamma_{N_{2}}^{2} M_{N_{2}}^{2}} \\
+\frac{\Gamma_{N_{1}} M_{N_{1}} \pi \delta\left(q^{2}-M_{N_{2}}^{2}\right)}{2\left[\left(M_{N_{2}}^{2}-M_{N_{1}}^{2}\right)^{2}+\Gamma_{N_{1}}^{2} M_{N_{1}}^{2}\right]} \\
+\frac{\Gamma_{N_{2}} M_{N_{2}} \pi \delta\left(q^{2}-M_{N_{1}}^{2}\right)}{2\left[\left(M_{N_{1}}^{2}-M_{N_{2}}^{2}\right)^{2}+\Gamma_{N_{2}}^{2} M_{N_{2}}^{2}\right]} .
\end{gathered}
$$

Finally, we can write the decay width as

$$
\begin{aligned}
d \Gamma^{\mathrm{LNV}}= & \left(1-\frac{\delta_{i j}}{2}\right) \frac{1}{2 m} \frac{\kappa}{(2 \pi)^{5}} \frac{\pi}{2} \frac{\pi}{4} d \cos \theta \\
& \times\left(\sum_{a=1}^{2}\left[\frac{\pi M_{N_{a}}}{\Gamma_{N_{a}}}\left|K_{R a i}\right|^{2}\left|K_{R a j}\right|^{2} T r_{21} \lambda^{1 / 2}\left(1, \frac{m_{i}^{2}}{m^{2}}, \frac{M_{N_{a}}^{2}}{m^{2}}\right) \lambda^{1 / 2}\left(1, \frac{m_{j}^{2}}{M_{N_{a}}^{2}}, \frac{m_{3}^{2}}{M_{N_{a}}^{2}}\right)\right]\right. \\
& +\pi M_{N} \operatorname{Re}\left[K_{R 1 i} K_{R 1 j} K_{R 2 i}^{*} K_{R 2 j}^{*}\left(\frac{1-4 i \frac{\delta M}{\Gamma_{N_{1}}}}{\Gamma_{N_{1}}\left(1+4 \frac{\delta M^{2}}{\Gamma_{N_{1}}^{2}}\right)}+\frac{1-4 i \frac{\delta M}{\Gamma_{N_{2}}}}{\Gamma_{N_{2}}\left(1+4 \frac{\delta M^{2}}{\Gamma_{N_{2}}^{2}}\right.}\right)\right] T r_{21} \\
& \left.\times \lambda^{1 / 2}\left(1, \frac{m_{i}^{2}}{m^{2}}, \frac{M_{N}^{2}}{m^{2}}\right) \lambda^{1 / 2}\left(1, \frac{m_{j}^{2}}{M_{N}^{2}}, \frac{m_{3}^{2}}{M_{N}^{2}}\right)+(i \leftrightarrow j)\right) .
\end{aligned}
$$

\section{LNC}

Following a similar procedure, the decay width for opposite-sign leptons can be calculated as

$$
\begin{aligned}
\mathcal{M}_{i j}^{\mathrm{LNC}, a}= & 2 G_{F}^{2} V_{M_{1}}^{\mathrm{CKM}} V_{M_{2}}^{\mathrm{CKM}} f_{M_{1}} f_{M_{2}}\left(\frac{M_{W_{L}}}{M_{W_{R}}}\right)^{4}\left(K_{R}\right)_{a i}\left(K_{R}^{*}\right)_{a j} \\
& \times \frac{\bar{u}\left(k_{1}\right) \not \not \phi q k_{3} P_{R} v\left(k_{2}\right)}{q^{2}-M_{N_{a}}^{2}+i \Gamma_{N_{a}} M_{N_{a}}}, \\
\left|\mathcal{M}_{i j}^{\mathrm{LNC}, a}\right|^{2}= & \kappa\left(K_{R}^{*}\right)_{a i}\left(K_{R}\right)_{a j}\left(K_{R}\right)_{a i}\left(K_{R}^{*}\right)_{a j} \\
& \times \frac{\operatorname{Tr}\left[\left(\not k_{2}-m_{2}\right) \not k_{3} \not \not p p P_{R}\left(\not k_{1}+m_{1}\right) \not p \not k_{3} P_{R}\right]}{\left(q^{2}-M_{N_{a}}^{2}\right)^{2}+\Gamma_{N_{a}}^{2} M_{N_{a}}^{2}},
\end{aligned}
$$

where

$$
\begin{aligned}
\operatorname{Tr}_{21}^{\mathrm{LNC}}= & \operatorname{Tr}\left[\left(\not k_{2}-m_{2}\right) \not k_{3} \not \not p P_{R}\left(\not k_{1}+m_{1}\right) \not p q k_{3} P_{R}\right] \\
= & 4 k_{1} \cdot q k_{2} \cdot q k_{3} \cdot k_{3} p \cdot p-8 k_{1} \cdot q k_{2} \cdot k_{3} k_{3} \cdot q p \cdot p \\
& -8 k_{1} \cdot p k_{2} \cdot q k_{3} \cdot k_{3} p \cdot q+16 k_{1} \cdot p k_{2} \cdot k_{3} k_{3} \cdot q p \cdot q \\
& +4 k_{1} \cdot p k_{2} \cdot p k_{3} \cdot k_{3} q \cdot q-8 k 1 \cdot p k_{2} \cdot k_{3} k_{3} \cdot p q \cdot q \\
& +4 k_{1} \cdot k_{3} k_{2} \cdot k_{3} p \cdot p q \cdot q-2 k_{1} \cdot k_{2} k_{3} \cdot k_{3} p \cdot p q \cdot q .
\end{aligned}
$$

We can write the LNC decay width as 


$$
\begin{aligned}
d \Gamma^{\mathrm{LNC}}= & \frac{1}{2 m} \frac{\kappa}{(2 \pi)^{5}} \frac{\pi}{2} \frac{\pi}{4} d \cos \theta \\
& \times \sum_{a=1}^{2}\left[\frac{\pi}{\Gamma_{N_{a}} M_{N_{a}}}\left|K_{R a i}\right|^{2}\left|K_{R a j}^{*}\right|^{2} \operatorname{Tr}_{21}^{\mathrm{LNC}} \lambda^{1 / 2}\left(1, \frac{m_{i}^{2}}{m^{2}}, \frac{M_{N_{a}}^{2}}{m^{2}}\right) \lambda^{1 / 2}\left(1, \frac{m_{j}^{2}}{M_{N_{a}}^{2}}, \frac{m_{3}^{2}}{M_{N_{a}}^{2}}\right)\right] \\
& +\frac{\pi}{M_{N}} \operatorname{Re}\left[K_{R 1 i}^{*} K_{R 1 j} K_{R 2 i} K_{R 2 j}^{*}\left(\frac{1-4 i \frac{\delta M}{\Gamma_{N_{1}}}}{\Gamma_{N_{1}}\left(1+4 \frac{\delta M^{2}}{\Gamma_{N_{1}}^{2}}\right)}+\frac{1-4 i \frac{\delta M}{\Gamma_{N_{2}}}}{\Gamma_{N_{2}}\left(1+4 \frac{\delta M^{2}}{\Gamma_{N_{2}}^{2}}\right)}\right)\right] T r_{21}^{\mathrm{LNC}} \\
& \times \lambda^{1 / 2}\left(1, \frac{m_{i}^{2}}{m^{2}}, \frac{M_{N}^{2}}{m^{2}}\right) \lambda^{1 / 2}\left(1, \frac{m_{j}^{2}}{M_{N}^{2}}, \frac{m_{3}^{2}}{M_{N}^{2}}\right) .
\end{aligned}
$$

\section{APPENDIX B: $\boldsymbol{R}_{e e}$ AND $\tilde{\boldsymbol{R}}_{e \mu}$}

$$
\begin{gathered}
R_{e e}=\frac{\sin ^{2} 2 \theta \sin \alpha_{1}\left(\sin \alpha_{1}-4 x \cos \alpha_{1}\right)}{\left(1+4 x^{2}\right)\left(2 \cos ^{4} \theta+2 \sin ^{4} \theta\right)+\sin ^{2} 2 \theta\left(\cos ^{2} \alpha_{1}+2 x \sin \alpha_{1}\right)}, \\
\tilde{R}_{e \mu}=\frac{\left(1+4 x^{2}\right)(1+3 \cos 4 \theta)+6 \sin ^{2} 2 \theta(\cos 2 \alpha+4 x \sin 2 \alpha)}{\left(1+4 x^{2}\right)(5-\cos 4 \theta)-2 \sin ^{2} 2 \theta(\cos 2 \alpha+4 x \sin 2 \alpha)} .
\end{gathered}
$$

\section{APPENDIX C: TOTAL DECAY WIDTH OF HEAVY MAJORANA NEUTRINO $N_{j}$}

Here we present the analytic expression of the total decay width of $N_{j}$ for our chosen mass range $0.14 \mathrm{GeV} \leq M_{N_{j}} \leq 6 \mathrm{GeV}$. In addition to the $\mathrm{SM}$ gauge bosons $W_{L}$ and $Z$, the gauge bosons $W_{R}$ and $Z^{\prime}$ will also contribute in the decays of RH neutrinos via chargedcurrent and neutral-current interactions. The analytical expressions for different two- and three-body partial decay widths of the RH neutrinos $N_{i}$ are given as follows.

\section{Two-body decays of $N$}

$$
\begin{aligned}
\Gamma\left(N_{j} \rightarrow \ell_{i}^{-} P^{+}\right)= & \frac{G_{F}^{2} M_{N_{j}}^{3}}{16 \pi} f_{p}^{2}\left|V_{q \bar{q}^{\prime}}\right|^{2}\left(\left|S_{i j}\right|^{2} F_{P}\left(x_{\ell_{i}}, x_{P}\right)\right. \\
& +\left|K_{R_{i j}}\right|^{2} \xi_{1}^{4} F_{P}\left(x_{\ell_{i}}, x_{P}\right) \\
& \left.+4 \operatorname{Re}\left[S_{i j} K_{R_{i j}}\right] \xi_{1}^{2} x_{\ell_{i}} x_{P}^{2} \lambda^{\frac{1}{2}}\left(1, x_{\ell_{i}}^{2}, x_{P}^{2}\right)\right),
\end{aligned}
$$

where $\ell_{1}=e, \ell_{2}=\mu, \ell_{3}=\tau$, and $P^{+}=\pi^{+}, K^{+}, D^{+}, D_{s}^{+}$.

$$
\begin{aligned}
\Gamma\left(N_{j} \rightarrow \ell_{i}^{-} V^{+}\right)= & \frac{G_{F}^{2} M_{N_{j}}^{3}}{16 \pi} f_{V}^{2}\left|V_{q \bar{q}^{\prime}}\right|^{2}\left(\left|S_{i j}\right|^{2} F_{V}\left(x_{\ell_{i}}, x_{V}\right)\right. \\
& +\mid K_{R_{i j}}{ }^{2} \xi_{1}^{4} F_{V}\left(x_{\ell_{i}}, x_{V}\right) \\
& \left.-12 \operatorname{Re}\left[S_{i j} K_{R_{i j}}\right] \xi_{1}^{2} x_{\ell_{i}} x_{V}^{2} \lambda^{\frac{1}{2}}\left(1, x_{\ell_{i}}^{2}, x_{V}^{2}\right)\right),
\end{aligned}
$$

where $\ell_{1}=e, \ell_{2}=\mu, \ell_{3}=\tau, \quad$ and $\quad V^{+}=\rho^{+}, K^{*+}$, $D^{*+}, D_{s}^{*+}$.

$$
\begin{array}{r}
\Gamma\left(N_{j} \rightarrow \nu_{\ell_{i}} P^{0}\right)=\frac{G_{F}^{2} M_{N_{j}}^{3}}{4 \pi} f_{P}^{2} \sum_{i}\left|U_{i j}\right|^{2}\left|S_{i j}\right|^{2} \\
\quad \times\left(K_{P}^{2}+K_{P}^{\prime 2} \xi_{2}^{4}-2 K_{P} K_{P}^{\prime} \xi_{2}^{2}\right) F_{P}\left(x_{\nu_{\ell}}, x_{P}\right),
\end{array}
$$

where $\nu_{\ell_{i}}$ are the flavor eigenstates $\nu_{e}, \nu_{\mu}, \nu_{\tau}$ and $P^{0}=\pi^{0}, \eta, \eta^{\prime}, \eta_{c}$.

$$
\begin{array}{r}
\Gamma\left(N_{j} \rightarrow \nu_{\ell_{i}} V^{0}\right)=\frac{G_{F}^{2} M_{N_{j}}^{3}}{4 \pi} f_{V}^{2} \sum_{i}\left|U_{i j}\right|^{2}\left|S_{i j}\right|^{2} \\
\times\left(K_{V}^{2}+K_{V}^{\prime 2} \xi_{2}^{4}-2 K_{V} K_{V}^{\prime} \xi_{2}^{2}\right) F_{V}\left(x_{\nu_{\ell}}, x_{P}\right),
\end{array}
$$

where $\nu_{\ell}=\nu_{e}, \nu_{\mu}, \nu_{\tau}$ and $V^{0}=\rho^{0}, \omega, \phi, J / \psi$. In the above, $\xi_{1}=\frac{M_{W_{L}}}{M_{W_{R}}}, \quad \xi_{2}=\frac{M_{Z}}{M_{Z^{\prime}}}, \quad x_{i}=\frac{m_{i}}{M_{N}}$, with $m_{i}=m_{\ell}, m_{P^{0}}, m_{V^{0}}$, $m_{P^{+}}, m_{V}^{+}$. The kinematical functions required for two-body decay are given by 


$$
\begin{aligned}
& F_{P}(x, y)=\left(\left(1+x^{2}\right)\left(1+x^{2}-y^{2}\right)-4 x^{2}\right) \lambda^{\frac{1}{2}}\left(1, x^{2}, y^{2}\right) \\
& F_{V}(x, y)=\left(\left(1-x^{2}\right)^{2}+\left(1+x^{2}\right) y^{2}-2 y^{4}\right) \lambda^{\frac{1}{2}}\left(1, x^{2}, y^{2}\right) .
\end{aligned}
$$

The neutral-current couplings of vector mesons are given by

The neutral-current couplings of pseudoscalar mesons are given by

$$
\begin{aligned}
K_{\pi^{0}} & =-\frac{1}{2 \sqrt{2}}, & K_{\pi^{0}}^{\prime} & =\frac{1}{\sqrt{2}}\left(\frac{1}{2}-\sin ^{2} \theta_{w}\right), \\
K_{\eta} & =-\frac{1}{2 \sqrt{6}}, & K_{\eta}^{\prime} & =\frac{1}{\sqrt{6}}\left(\frac{1}{2}-\sin ^{2} \theta_{w}\right), \\
K_{\eta^{\prime}} & =\frac{1}{4 \sqrt{3}}, & K_{\eta^{\prime}}^{\prime} & =\frac{1}{\sqrt{3}}\left(-\frac{1}{4}+\frac{1}{2} \sin ^{2} \theta_{w}\right), \\
K_{\eta_{c}} & =-\frac{1}{4}, & K_{\eta_{c}}^{\prime} & =\left(\frac{1}{4}-\frac{1}{2} \sin ^{2} \theta_{w}\right) .
\end{aligned}
$$

$$
\begin{aligned}
K_{\rho^{0}} & =\frac{1}{\sqrt{2}}\left(\frac{1}{2}-\sin ^{2} \theta_{w}\right), \\
K_{\omega} & =-\frac{1}{3 \sqrt{2}} \sin ^{2} \theta_{w}, \\
K_{\phi} & =\left(-\frac{1}{4}+\frac{1}{3} \sin ^{2} \theta_{w}\right), \\
K_{J / \psi} & =\left(\frac{1}{4}-\frac{2}{3} \sin ^{2} \theta_{w}\right) .
\end{aligned}
$$

\section{Three-body decays of $N$}

$$
\begin{aligned}
\Gamma\left(N_{j} \rightarrow \ell_{i}^{-} \ell_{k}^{+} \nu_{\ell_{k}}\right)= & \frac{G_{F}^{2} M_{N_{j}}^{5}}{16 \pi^{3}}\left(\left|S_{i j}\right|^{2} \sum_{\kappa}\left|U_{k \kappa}\right|^{2} I_{1}\left(x_{\ell_{i}}, x_{\nu_{\ell_{k}}}, x_{\ell_{k}}\right)+\left|K_{R_{i j}}\right|^{2}\right. \\
& \left.\times \sum_{\kappa}\left|T_{k \kappa}\right|^{2} \xi_{1}^{4} I_{1}\left(x_{\ell_{i}}, x_{\nu_{\ell_{k}}}, x_{\ell_{k}}\right)-8 \operatorname{Re}\left(S_{i j}^{*} V_{i j}^{*} \sum_{\kappa} U_{k \kappa} T_{k \kappa}\right) \xi_{1}^{2} I_{3}\left(x_{\ell_{i}}, x_{\nu_{\ell_{k}}}, x_{\ell_{k}}\right)\right),
\end{aligned}
$$

where $\ell_{i}, \ell_{j}=e, \mu, \tau, \ell_{i} \neq \ell_{j}$.

$$
\begin{aligned}
\Gamma\left(N_{j} \rightarrow \nu_{\ell_{i}} \ell_{i}^{-} \ell_{i}^{+}\right)= & \frac{G_{F}^{2} M_{N_{j}}^{5}}{16 \pi^{3}}\left(| S _ { i j } | ^ { 2 } \sum _ { k } | U _ { i k } | ^ { 2 } \left[I_{1}\left(x_{\nu_{\ell_{i}}}, x_{\ell_{i}}, x_{\ell_{i}}\right)\right.\right. \\
& +2\left(\left(g_{V}^{\ell}\right)^{2}+\left(g_{A}^{\ell}\right)^{2}\right) I_{1}\left(x_{\nu_{\ell_{i}}}, x_{\ell_{i}}, x_{\ell_{i}}\right)+2\left(\left(g_{V}^{\ell}\right)^{2}-\left(g_{A}^{\ell}\right)^{2}\right) I_{2}\left(x_{\nu_{\ell_{i}}}, x_{\ell_{i}}, x_{\ell_{i}}\right) \\
& +2\left(\left(g_{V}^{\ell}\right)^{2}+\left(g_{A}^{\prime \ell}\right)^{2}\right) \xi_{2}^{4} I_{1}\left(x_{\nu_{\ell_{i}}}, x_{\ell_{i}}, x_{\ell_{i}}\right)+2\left(\left(g_{V}^{\prime \ell}\right)^{2}-\left(g_{A}^{\prime \ell}\right)^{2}\right) \xi_{2}^{4} I_{2}\left(x_{\nu_{\ell_{i}}}, x_{\ell_{i}}, x_{\ell_{i}}\right) \\
& \left.-4 \xi_{2}^{2}\left(\left(g_{V}^{\ell} g_{V}^{\ell}+g_{A}^{\ell} g_{A}^{\prime \ell}\right) I_{1}\left(x_{\nu_{\ell_{i}}}, x_{\ell_{i}}, x_{\ell_{i}}\right)+\left(g_{V}^{\ell} g_{V}^{\prime \ell}-g_{A}^{\ell} g_{A}^{\prime \ell}\right) I_{2}\left(x_{\nu_{\ell_{i}}}, x_{\ell_{i}}, x_{\ell_{i}}\right)\right)\right] \\
& +\left|V_{i j}\right|^{2} \sum_{k}\left|T_{i k}\right|^{2} \xi_{1}^{4} I_{1}\left(x_{\nu_{\ell_{i}}}, x_{\ell_{i}}, x_{\ell_{i}}\right)-8 \operatorname{Re}\left[S_{i j}^{*} V_{i j}^{*} \sum_{k} U_{i k} T_{i k}\right] \xi_{1}^{2} I_{3}\left(x_{\ell_{i}}, x_{\nu_{\ell_{i}}}, x_{\ell_{i}}\right) \\
& +2 \operatorname{Re}\left[\left|S_{i j}\right|^{2} \sum_{k}\left|U_{i k}\right|^{2}\right]\left[\xi_{2}^{2}\left(g_{A}^{\ell \ell}-g_{V}^{\prime \ell}\right) I_{1}\left(x_{\nu_{\ell_{i}}}, x_{\ell_{i}}, x_{\ell_{i}}\right)-\xi_{2}^{2}\left(g_{A}^{\prime \ell}+g_{V}^{\ell}\right) I_{2}\left(x_{\nu_{\ell_{i}}}, x_{\ell_{i}}, x_{\ell_{i}}\right)\right. \\
& \left.-\left(g_{A}^{\ell}-g_{V}^{\ell}\right) I_{1}\left(x_{\nu_{\ell_{i}}}, x_{\ell_{i}}, x_{\ell_{i}}\right)+\left(g_{A}^{\ell}+g_{V}^{\ell}\right) I_{2}\left(x_{\nu_{\ell_{i}}}, x_{\ell_{i}}, x_{\ell_{i}}\right)\right] \\
& -8 \operatorname{Re}\left[S_{i j} V_{i j} \sum_{k} U_{i k}^{*} T_{i k}^{*}\right] \xi_{1}^{2}\left[\left(g_{V}^{\ell \ell}-g_{A}^{\prime \ell}\right) \xi_{2}^{2} I_{3}\left(x_{\nu_{\ell_{i}}}, x_{\ell_{i}}, x_{\ell_{i}}\right)+\frac{1}{4}\left(g_{V}^{\prime \ell}+g_{A}^{\prime \ell}\right) \xi_{2}^{2}\right. \\
& \left.\left.\times I_{4}\left(x_{\ell_{i}}, x_{\ell_{i}}, x_{\nu_{\ell_{i}}}\right)+\left(g_{V}^{\ell}-g_{A}^{\ell}\right) I_{3}\left(x_{\nu_{\ell_{i}}}, x_{\ell_{i}}, x_{\ell_{i}}\right)+\frac{1}{4}\left(g_{V}^{\ell}+g_{A}^{\ell}\right) I_{4}\left(x_{\ell_{i}}, x_{\ell_{i}}, x_{\nu_{\ell_{i}}}\right)\right]\right)
\end{aligned}
$$


where $\ell_{i}=e, \mu, \tau$.

$$
\begin{aligned}
\Gamma\left(N_{j} \rightarrow \nu_{\ell_{i}} \ell_{k}^{-} \ell_{k}^{+}\right)= & \frac{G_{F}^{2} M_{N_{j}}^{5}}{8 \pi^{3}}\left|S_{i j}\right|^{2} \sum_{k}\left|U_{i \kappa}\right|^{2}\left[\left(\left(g_{V}^{\ell}\right)^{2}+\left(g_{A}^{\ell}\right)^{2}\right) I_{1}\left(x_{\nu_{\ell_{i}}}, x_{\ell_{k}}, x_{\ell_{k}}\right)\right. \\
& +\left(\left(g_{V}^{\ell}\right)^{2}-\left(g_{A}^{\ell}\right)^{2}\right) I_{2}\left(x_{\nu_{\ell_{i}}}, x_{\ell_{k}}, x_{\ell_{k}}\right)+\left(\left(g_{V}^{\ell \ell}\right)^{2}+\left(g_{A}^{\prime \ell}\right)^{2}\right) \xi_{2}^{4} I_{1}\left(x_{\nu_{\ell_{i}}}, x_{\ell_{k}}, x_{\ell_{k}}\right) \\
& +\left(\left(g_{V}^{\prime \ell}\right)^{2}-\left(g_{A}^{\prime \ell}\right)^{2}\right) \xi_{2}^{4} I_{2}\left(x_{\nu_{\ell_{i}}}, x_{\ell_{k}}, x_{\ell_{k}}\right)-2 \xi_{2}^{2}\left[\left(g_{V}^{\ell} g_{V}^{\prime \ell}+g_{A}^{\ell} g_{A}^{\prime \ell}\right) I_{1}\left(x_{\nu_{\ell_{i}}}, x_{\ell_{k}}, x_{\ell_{k}}\right)\right. \\
& \left.\left.+\left(g_{V}^{\ell} g_{V}^{\prime \ell}-g_{A}^{\ell} g_{A}^{\ell \ell}\right) I_{2}\left(x_{\nu_{\ell_{i}}}, x_{\ell_{k}}, x_{\ell_{k}}\right)\right]\right],
\end{aligned}
$$

where $\ell_{i}, \ell_{j}=e, \mu, \tau$ and $\ell_{i} \neq \ell_{j}$.

$$
\Gamma\left(N_{j} \rightarrow \nu_{\ell_{i}} \nu \bar{\nu}\right)=\frac{G_{F}^{2} M_{N_{j}}^{5}}{192 \pi^{3}}\left|S_{i j}\right|^{2} \sum_{k}\left|U_{i k}\right|^{2}\left(1-\sin ^{2} \theta_{w} \xi_{2}^{2}\right)^{2} .
$$

The kinematical functions required for three-body decay are given by

$$
\begin{aligned}
& I_{1}(x, y, z)=\int_{(x+y)^{2}}^{(1-z)^{2}} \frac{d s}{s}\left(s-x^{2}-y^{2}\right)\left(1+z^{2}-s\right) \lambda^{\frac{1}{2}}\left(s, x^{2}, y^{2}\right) \lambda^{\frac{1}{2}}\left(1, s, z^{2}\right), \\
& I_{2}(x, y, z)=y z \int_{(y+z)^{2}}^{(1-x)^{2}} \frac{d s}{s}\left(1+x^{2}-s\right) \lambda^{\frac{1}{2}}\left(s, y^{2}, z^{2}\right) \lambda^{\frac{1}{2}}\left(1, s, x^{2}\right), \\
& I_{3}(x, y, z)=x y z \int_{(x+y)^{2}}^{(1-z)^{2}} \frac{d s}{s} \lambda^{\frac{1}{2}}\left(s, x^{2}, y^{2}\right) \lambda^{\frac{1}{2}}\left(1, s, z^{2}\right), \\
& I_{4}(x, y, z)=z \int_{(x+y)^{2}}^{(1-z)^{2}} \frac{d s}{s} \lambda^{\frac{1}{2}}\left(s, x^{2}, y^{2}\right) \lambda^{\frac{1}{2}}\left(1, s, z^{2}\right) .
\end{aligned}
$$

The neutral-current couplings of leptons are given by

$$
\begin{array}{ll}
g_{V}^{\ell}=-\frac{1}{4}+\sin ^{2} \theta_{w}, & g_{A}^{\ell}=\frac{1}{4}, \\
g_{V}^{\prime \ell}=-\frac{1}{4}+\sin ^{2} \theta_{w}, & g_{A}^{\prime \ell}=-\frac{1}{4}+\frac{1}{2} \sin ^{2} \theta_{w} .
\end{array}
$$

The functions $A\left(M_{N}\right)$ and $B\left(M_{N}\right)$, relevant for the $N \rightarrow l \pi$ decay mode, are given by

$$
\begin{aligned}
& A\left(M_{N}\right)=\frac{G_{F}^{2} M_{N}^{3}}{16 \pi} f_{\pi}^{2} V_{u d}^{2} \xi_{1}^{4}\left[\frac{F_{P}\left(x_{e}, x_{\pi}\right)+F_{P}\left(x_{\mu}, x_{\pi}\right)}{2}\right], \\
& B\left(M_{N}\right)=\frac{G_{F}^{2} M_{N}^{3}}{16 \pi} f_{\pi}^{2} V_{u d}^{2} \xi_{1}^{4}\left[\frac{F_{P}\left(x_{e}, x_{\pi}\right)-F_{P}\left(x_{\mu}, x_{\pi}\right)}{2}\right] .
\end{aligned}
$$

[1] J. C. Pati and A. Salam, Lepton number as the fourth color, Phys. Rev. D 10, 275 (1974).

[2] M. Agostini et al. (GERDA Collaboration), Improved Limit on Neutrinoless Double- $\beta$ Decay of ${ }^{76} \mathrm{Ge}$ from GERDA Phase II, Phys. Rev. Lett. 120, 132503 (2018).

[3] G. Racah, On the symmetry of particle and antiparticle, Nuovo Cimento 14, 322 (1937). 
[4] W. Furry, On transition probabilities in double betadisintegration, Phys. Rev. 56, 1184 (1939).

[5] J. Schechter and J. Valle, Neutrinoless double beta decay in $\mathrm{SU}(2) \times \mathrm{U}(1)$ theories, Phys. Rev. D 25, 2951 (1982).

[6] V. Tello, M. Nemevsek, F. Nesti, G. Senjanovic, and F. Vissani, Left-Right Symmetry: From LHC to Neutrinoless Double Beta Decay, Phys. Rev. Lett. 106, 151801 (2011).

[7] P. S. B. Dev, S. Goswami, and M. Mitra, TeV scale left-right symmetry and large mixing effects in neutrinoless double beta decay, Phys. Rev. D 91, 113004 (2015).

[8] W.-Y. Keung and G. Senjanovic, Majorana Neutrinos and the Production of the Right-handed Charged Gauge Boson, Phys. Rev. Lett. 50, 1427 (1983).

[9] C.-Y. Chen, P. S. B. Dev, and R. Mohapatra, Probing heavylight neutrino mixing in left-right seesaw models at the LHC, Phys. Rev. D 88, 033014 (2013).

[10] M. Mitra, R. Ruiz, D. J. Scott, and M. Spannowsky, Neutrino jets from high-mass $W_{R}$ gauge bosons in TeVscale left-right symmetric models, Phys. Rev. D 94, 095016 (2016).

[11] M. Nemevšek, F. Nesti, and G. Popara, Keung-Senjanović process at the LHC: From lepton number violation to displaced vertices to invisible decays, Phys. Rev. D 97, 115018 (2018).

[12] M. Nemevšek, F. Nesti, and J. C. Vasquez, Majorana Higgses at colliders, J. High Energy Phys. 04 (2017) 114.

[13] S. Mandal, Search for sterile neutrinos at colliders, Ph.D thesis, HBNI, Mumbai, 2019, https://www.imsc.res.in/ xmlui/bitstream/handle/123456789/445/HBNI\%20Th157 .pdf? sequence $=1 \&$ is Allowed $=y$.

[14] A. Atre, T. Han, S. Pascoli, and B. Zhang, The search for heavy majorana neutrinos, J. High Energy Phys. 05 (2009) 030 .

[15] R. Aaij et al. (LHCb Collaboration), Search for Majorana Neutrinos in $B^{-} \rightarrow \pi^{+} \mu^{-} \mu^{-}$Decays, Phys. Rev. Lett. 112, 131802 (2014).

[16] G. Cvetic, C. Dib, S. K. Kang, and C. Kim, Probing Majorana neutrinos in rare $\mathrm{K}$ and $\mathrm{D}, D_{s}, \mathrm{~B}, B_{c}$ meson decays, Phys. Rev. D 82, 053010 (2010).

[17] G. Cvetic, C. Kim, S. Mendizabal, and J. Zamora-Saa, Exploring $C P$-violation, via heavy neutrino oscillations, in rare B meson decays at Belle II, Eur. Phys. J. C 80, 1052 (2020).

[18] B. Shuve and M. E. Peskin, Revision of the LHCb Limit on Majorana Neutrinos, Phys. Rev. D 94, 113007 (2016).

[19] E. J. Chun, A. Das, S. Mandal, M. Mitra, and N. Sinha, Sensitivity of lepton number violating meson decays in different experiments, Phys. Rev. D 100, 095022 (2019).

[20] S. Mandal and N. Sinha, Favoured $B_{c}$ decay modes to search for a Majorana neutrino, Phys. Rev. D 94, 033001 (2016).

[21] S. Mandal, M. Mitra, and N. Sinha, Constraining the righthanded gauge boson mass from lepton number violating meson decays in a low scale left-right model, Phys. Rev. D 96, 035023 (2017).

[22] D. Milanes, N. Quintero, and C.E. Vera, Sensitivity to Majorana neutrinos in $\Delta L=2$ decays of $B_{c}$ meson at LHCb, Phys. Rev. D 93, 094026 (2016).
[23] A. Abada, V. De Romeri, M. Lucente, A. M. Teixeira, and T. Toma, Effective Majorana mass matrix from tau and pseudoscalar meson lepton number violating decays, J. High Energy Phys. 02 (2018) 169.

[24] J. C. Helo, S. Kovalenko, and I. Schmidt, Sterile neutrinos in lepton number and lepton flavor violating decays, Nucl. Phys. B853, 80 (2011).

[25] A. Abada, C. Hati, X. Marcano, and A. Teixeira, Interference effects in LNV and LFV semileptonic decays: The Majorana hypothesis, J. High Energy Phys. 09 (2019) 017.

[26] M. Aaboud et al. (ATLAS Collaboration), Search for heavy Majorana or Dirac neutrinos and right-handed $W$ gauge bosons in final states with two charged leptons and two jets at $\sqrt{s}=13 \mathrm{TeV}$ with the ATLAS detector, J. High Energy Phys. 01 (2019) 016.

[27] A. M. Sirunyan et al. (CMS Collaboration), Search for a heavy right-handed $\mathrm{W}$ boson and a heavy neutrino in events with two same-flavor leptons and two jets at $\sqrt{s}=13 \mathrm{TeV}$, J. High Energy Phys. 05 (2018) 148.

[28] M. Aaboud et al. (ATLAS Collaboration), Search for a right-handed gauge boson decaying into a high-momentum heavy neutrino and a charged lepton in $p p$ collisions with the ATLAS detector at $\sqrt{s}=13 \mathrm{TeV}$, Phys. Lett. B 798, 134942 (2019).

[29] T. Han, I. Lewis, R. Ruiz, and Z.-G. Si, Lepton number violation and $W^{\prime}$ chiral couplings at the LHC, Phys. Rev. D 87, 035011 (2013).

[30] R. Ruiz, A quantitative study on helicity inversion in Majorana neutrino decays at the LHC, Phys. Rev. D 103, 015022 (2021).

[31] M. Lindner, F. S. Queiroz, and W. Rodejohann, Dilepton bounds on left-right symmetry at the LHC run II and neutrinoless double beta decay, Phys. Lett. B 762, 190 (2016).

[32] M. Hirsch and Z.S. Wang, Heavy neutral leptons at ANUBIS, Phys. Rev. D 101, 055034 (2020).

[33] G. Cvetič, C. S. Kim, and J. Zamora-Saá, $C P$ violations in $\pi^{ \pm}$Meson Decay, J. Phys. G 41, 075004 (2014).

[34] G. Cvetic, C. Dib, C. S. Kim, and J. Zamora-Saa, Probing the Majorana neutrinos and their $C P$ violation in decays of charged scalar mesons $\pi, K, D, D_{s}, B, B_{c}$, Symmetry 7, 726 (2015).

[35] G. Cvetic, C.S. Kim, R. Kogerler, and J. Zamora-Saa, Oscillation of heavy sterile neutrino in decay of $B \rightarrow \mu e \pi$, Phys. Rev. D 92, 013015 (2015).

[36] G. Moreno and J. Zamora-Saa, Rare meson decays with three pairs of quasi-degenerate heavy neutrinos, Phys. Rev. D 94, 093005 (2016).

[37] J. Zamora-Saa, Resonant $C P$ violation in rare $\tau^{ \pm}$decays, J. High Energy Phys. 05 (2017) 110.

[38] G. Cvetič, A. Das, and J. Zamora-Saá, Probing heavy neutrino oscillations in rare $W$ boson decays, J. Phys. G 46, 075002 (2019).

[39] G. Cvetič, A. Das, S. Tapia, and J. Zamora-Saá, Measuring the heavy neutrino oscillations in rare $\mathrm{W}$ boson decays at the Large Hadron Collider, J. Phys. G 47, 015001 (2020).

[40] S. Tapia and J. Zamora-Saá, Exploring $C P$-Violating heavy neutrino oscillations in rare tau decays at Belle II, Nucl. Phys. B952, 114936 (2020). 
[41] J. Gluza and T. Jeliński, Heavy neutrinos and the $p p \rightarrow l l j j$ CMS data, Phys. Lett. B 748, 125 (2015).

[42] A. Das, P. S. B. Dev, and R. N. Mohapatra, Same sign versus opposite sign dileptons as a probe of low scale seesaw mechanisms, Phys. Rev. D 97, 015018 (2018).

[43] S. Bray, J. S. Lee, and A. Pilaftsis, Resonant $C P$ violation due to heavy neutrinos at the LHC, Nucl. Phys. B786, 95 (2007).

[44] A. Pilaftsis, Resonant $C P$ violation induced by particle mixing in transition amplitudes, Nucl. Phys. B504, 61 (1997).

[45] J. Gunion, R. Vega, and J. Wudka, Higgs triplets in the Standard Model, Phys. Rev. D 42, 1673 (1990).

[46] A. Maiezza, M. Nemevsek, F. Nesti, and G. Senjanovic, Left-right symmetry at LHC, Phys. Rev. D 82, 055022 (2010).

[47] G. Senjanović and V. Tello, Right Handed Quark Mixing in Left-Right Symmetric Theory, Phys. Rev. Lett. 114, 071801 (2015).

[48] R. N. Mohapatra and D. P. Sidhu, Gauge theories of weak interactions with left-right symmetry and the structure of neutral currents, Phys. Rev. D 16, 2843 (1977).

[49] V. Tello, Connections between the high and low energy violation of Lepton and Flavor numbers in the minimal leftright symmetric model, Ph.D thesis, SISSA, Trieste, 2012.
[50] Z.-H. Li, Z.-G. Si, Y. Wang, and N. Zhu, $B \rightarrow \pi \ell^{+} \ell^{-}$ decays revisited in the standard model, arXiv:1411.0466.

[51] L. Littenberg and G. Valencia, Rare and radiative kaon decays, Annu. Rev. Nucl. Part. Sci. 43, 729 (1993).

[52] V. Cirigliano, G. Ecker, H. Neufeld, A. Pich, and J. Portoles, Kaon decays in the Standard Model, Rev. Mod. Phys. 84, 399 (2012).

[53] A. Dolgov, S. Hansen, G. Raffelt, and D. Semikoz, Heavy sterile neutrinos: Bounds from big bang nucleosynthesis and SN1987A, Nucl. Phys. B590, 562 (2000).

[54] M. Kawasaki, K. Kohri, and T. Moroi, Big-Bang nucleosynthesis and hadronic decay of long-lived massive particles, Phys. Rev. D 71, 083502 (2005).

[55] M. Kawasaki, K. Kohri, T. Moroi, and Y. Takaesu, Revisiting big-bang nucleosynthesis constraints on long-lived decaying particles, Phys. Rev. D 97, 023502 (2018).

[56] M. Tanabashi et al. (Particle Data Group Collaboration), Review of particle physics, Phys. Rev. D 98, 030001 (2018).

[57] P. de Salas, D. Forero, S. Gariazzo, P. Martínez-Miravé, O. Mena, C. Ternes, M. Tórtola, and J. Valle, 2020 Global reassessment of the neutrino oscillation picture, J. High Energy Phys. 02 (2021) 071. 